\title{
Marriage Penalty: Unconditional Quantile Regression of Housework Participation in Japan
}

\author{
Kamila Kolpashnikova, University of Oxford \\ Man Yee Kan, University of Oxford \\ Kiyomi Shirakawa, Hitotsubashi University
}

\begin{abstract}
We analyze cross-sectional time-use diaries from the 2011 and 2016 Survey on Time Use and Leisure Activities (Shakai Seikatsu Kihon Chosa) to investigate the association between educational level and housework participation at different quantiles. Using the unconditional quantile regression method, we test whether education is associated with less time spent on housework as the previous research on highly educated people suggests. We find that this hypothesis stands only for non-married Japanese women. On the other hand, among married Japanese women, especially those without children in higher deciles of housework participation, are more likely to increase their participation in housework with the increase in their educational level. The results suggest that in Japan, the institute of marriage places higher expectations on women's housework participation on married women with higher levels of education.
\end{abstract}

Keywords: gender, housework, Japanese households, routine housework, unconditional quantile regression

\section{Introduction}

In this discussion paper, we investigate whether educational level can predict the patterns of participation in the domestic labor uniformly across different levels of participation in housework, and on different life-course stages. The main purpose of this study is to explore whether higher educational attainment is associated with lower housework participation among Japanese women. We also analyze whether the association of education at different deciles of housework participation among Japanese women varies at different life stages. Thus, we compare patterns for non-married women, married women without children, and married women with children. Our primary contribution is two-fold. First, we show that marriage is still a barrier 
to women in reducing their housework, regardless of their educational level. Second, we contribute to housework research by applying unconditional quantile regression method (Firpo, Fortin, \& Lemieux, 2009) to the analysis of housework participation. The study addresses two principal research questions: (1) How is educational attainment associated with different levels of housework participation? (2) whether the patterns are similar among non-married and married women, as well as women with and without children.

\section{Theoretical Expectations}

Education is one of the individual resources. Moreover, it reflects the gender socialization as a result of the acquired educational level. According to the expectations of the resource-based housework theories, highly educated women are more likely to be employed and thus have less time on housework, compared to women with lower levels of education (England, Garcia-Beaulieu, \& Ross, 2004; Kolpashnikova, 2018; Rubery, Smith, \& Fagan, 1999).

Following the resource-based housework participation explanation, we expect that:

Hypothesis 1: On all levels of housework participation the effects of education would be negatively associated with participation in housework.

Moreover, life-course transitions such as the transition from being single to being married are expected to have an effect on the participation in housework. For example, South and Spitze (1994) found that among both women and men, marriage was associated with more housework time. Marriage is also associated with more traditional gender attitudes (Zhou, 2017). On the 
other hand, Baxter, Hewitt, and Haynes (2008) found that the effects are applicable to women but not to men. They found that men's housework time remained considerably stable throughout life course stages. Thus, we expect the following:

Hypothesis 2: The higher the level of education, the less routine housework is performed but the association becomes weaker when women and men move across the life stages: from non-married to married, and from married with no children to married with dependent children.

\section{Data and Methods}

We employed microfiles of the 2011 and 2016 Survey on Time Use and Leisure Activities (STULA), also known as the Shakai Seikatsu Kihon Chosa. We accessed the microfiles at the data center of the Hitotsubashi University in Japan. The STULA collects time diaries for two consecutive days. This survey design allows treating the data as panel data, where the date of the diary is considered as a time variable. Time use diaries are more apt for measuring housework participation than the traditional 'stylized' surveys (Kan \& Pudney, 2008). The survey sample uses a two-stage stratified sampling method. The primary sampling unit is the enumeration district (ED) of the Population Census and the secondary unit is households. In 47 Japanese prefectures, a total of 6,902 EDs were selected. All household members 10 years of age and above were asked to complete the survey. Foreigners living in Japan were also included in the survey.

There are three main subsamples within models: non-married women without children (10,544 observations in 2011 and 9,699-in 2016), married women without children (24,543 observations in 2011 and 21,248 - in 2016), and married women with children (32,400 
observations in 2011 and 29,034 - in 2016). As the observation numbers show, the majority of women in the full sample reported to be married. We did not perform an analysis for nonmarried women with children because there are not enough observations in the Japanese dataset for the subsample. Moreover, we selected a sample of women who performed any housework on the diary day $(n=127,468)$ and personal weights were re-coded based on the original survey weights, scaled to the original sample size, where needed.

\section{Measures}

\section{Dependent Variables}

The dependent variable is represented by an aggregate measure of the time spent on indoor housework, such as cooking, cleaning, and doing the laundry. The participation in housework variables in all three countries is measured in minutes on a diary day.

Tables 1a-3b summarize the descriptive statistics for housework time among women in different deciles of housework participation in Japan. They show great differences among women in the top and bottom deciles of housework participation. Women in the top decile, on average, spend more than 10 times the time that women in the bottom decile spend on housework. Among all groups of women, women in the uppermost decile spend more than 500 minutes a day on housework, whereas women in the lowest decile spend about 40 minutes a day. The averages for women among the three groups show that married women spend substantially more time on housework than non-married women. For instance, non-married women who report doing housework on the diary day spend 133 minutes on housework on an average day in 2016, whereas married women with children-approximately 234 minutes. 
Table 1a Descriptive statistics, 20 to 60-year-old non-married women, Japan 2011.

\begin{tabular}{|c|c|c|c|c|c|c|c|c|c|c|c|}
\hline & $\begin{array}{l}\text { Women in } \\
2011 \\
1 \\
(0-10 \% \text { of } \\
\text { Housewor } \\
\text { k) }\end{array}$ & $\begin{array}{c}2 \\
(10-20 \% \\
\text { of } \\
\text { Housewor } \\
\text { k) } \\
\end{array}$ & $\begin{array}{c}3 \\
(20-30 \% \\
\text { of } \\
\text { Housewor } \\
\text { k) } \\
\end{array}$ & $\begin{array}{c}4 \\
(30-40 \% \\
\text { of } \\
\text { Housewor } \\
\text { k) } \\
\end{array}$ & $\begin{array}{c}5 \\
(40-50 \% \\
\text { of } \\
\text { Housewor } \\
\text { k) } \\
\end{array}$ & $\begin{array}{c}6 \\
(50-60 \% \\
\text { of } \\
\text { Housewor } \\
\text { k) }\end{array}$ & $\begin{array}{c}7 \\
(60-70 \% \\
\text { of } \\
\text { Housewor } \\
\text { k) } \\
\end{array}$ & $\begin{array}{c}8 \\
(70-80 \% \\
\text { of } \\
\text { Housewor } \\
\text { k) }\end{array}$ & $\begin{array}{c}9 \\
(80-90 \% \\
\text { of } \\
\text { Housewor } \\
\text { k) }\end{array}$ & $\begin{array}{c}10 \\
(90-100 \% \\
\text { of } \\
\text { Housewor } \\
\text { k) }\end{array}$ & Total \\
\hline Housework & $\begin{array}{c}39.883 \\
(16.203)\end{array}$ & $\begin{array}{l}82.793 \\
(7.497)\end{array}$ & $\begin{array}{l}113.330 \\
(7.457)\end{array}$ & $\begin{array}{l}142.872 \\
(7.495)\end{array}$ & $\begin{array}{l}173.360 \\
(7.456)\end{array}$ & $\begin{array}{l}208.168 \\
(11.742)\end{array}$ & $\begin{array}{l}253.555 \\
(12.546)\end{array}$ & $\begin{array}{l}298.942 \\
(11.282)\end{array}$ & $\begin{array}{l}361.370 \\
(25.208)\end{array}$ & $\begin{array}{l}516.259 \\
(92.046)\end{array}$ & $\begin{array}{c}131.705 \\
(112.335)\end{array}$ \\
\hline Education & $\begin{array}{l}13.256 \\
(1.918)\end{array}$ & $\begin{array}{l}13.068 \\
(1.971)\end{array}$ & $\begin{array}{l}12.943 \\
(1.912)\end{array}$ & $\begin{array}{l}12.800 \\
(1.910)\end{array}$ & $\begin{array}{l}12.881 \\
(1.998)\end{array}$ & $\begin{array}{l}12.866 \\
(1.911)\end{array}$ & $\begin{array}{l}12.774 \\
(2.080)\end{array}$ & $\begin{array}{l}12.791 \\
(1.864)\end{array}$ & $\begin{array}{l}12.806 \\
(1.983)\end{array}$ & $\begin{array}{l}12.362 \\
(1.947)\end{array}$ & $\begin{array}{l}13.017 \\
(1.951)\end{array}$ \\
\hline Paid Work Time & $\begin{array}{c}272.398 \\
(255.933)\end{array}$ & $\begin{array}{c}216.386 \\
(242.205)\end{array}$ & $\begin{array}{c}180.664 \\
(226.763)\end{array}$ & $\begin{array}{c}148.875 \\
(214.260)\end{array}$ & $\begin{array}{c}110.617 \\
(192.074)\end{array}$ & $\begin{array}{c}96.676 \\
(176.347)\end{array}$ & $\begin{array}{c}60.972 \\
(143.252)\end{array}$ & $\begin{array}{c}51.537 \\
(127.773)\end{array}$ & $\begin{array}{c}29.098 \\
(96.113)\end{array}$ & $\begin{array}{c}6.259 \\
(34.429)\end{array}$ & $\begin{array}{c}183.345 \\
(236.457)\end{array}$ \\
\hline Age & $\begin{array}{c}36.979 \\
(11.357)\end{array}$ & $\begin{array}{c}40.313 \\
(11.970)\end{array}$ & $\begin{array}{c}41.440 \\
(11.746)\end{array}$ & $\begin{array}{c}43.219 \\
(11.804)\end{array}$ & $\begin{array}{c}43.611 \\
(11.429)\end{array}$ & $\begin{array}{c}45.176 \\
(10.924)\end{array}$ & $\begin{array}{c}45.895 \\
(11.196)\end{array}$ & $\begin{array}{c}46.363 \\
(10.481)\end{array}$ & $\begin{array}{c}46.909 \\
(11.123)\end{array}$ & $\begin{array}{c}46.766 \\
(10.333)\end{array}$ & $\begin{array}{c}41.011 \\
(11.972)\end{array}$ \\
\hline Household size & $\begin{array}{c}2.831 \\
(1.484)\end{array}$ & $\begin{array}{c}2.611 \\
(1.464)\end{array}$ & $\begin{array}{c}2.631 \\
(1.449)\end{array}$ & $\begin{array}{c}2.601 \\
(1.399)\end{array}$ & $\begin{array}{c}2.650 \\
(1.430)\end{array}$ & $\begin{array}{c}2.659 \\
(1.345)\end{array}$ & $\begin{array}{c}2.688 \\
(1.426)\end{array}$ & $\begin{array}{c}2.746 \\
(1.308)\end{array}$ & $\begin{array}{c}2.817 \\
(1.394)\end{array}$ & $\begin{array}{c}2.879 \\
(1.201)\end{array}$ & $\begin{array}{c}2.725 \\
(1.440)\end{array}$ \\
\hline Employed & $\begin{array}{c}0.822 \\
(0.382)\end{array}$ & $\begin{array}{c}0.743 \\
(0.437)\end{array}$ & $\begin{array}{c}0.682 \\
(0.466)\end{array}$ & $\begin{array}{c}0.644 \\
(0.479)\end{array}$ & $\begin{array}{c}0.604 \\
(0.489)\end{array}$ & $\begin{array}{c}0.543 \\
(0.499)\end{array}$ & $\begin{array}{c}0.488 \\
(0.500)\end{array}$ & $\begin{array}{c}0.431 \\
(0.496)\end{array}$ & $\begin{array}{c}0.432 \\
(0.496)\end{array}$ & $\begin{array}{c}0.341 \\
(0.475)\end{array}$ & $\begin{array}{c}0.684 \\
(0.465)\end{array}$ \\
\hline Urban & $\begin{array}{c}0.881 \\
(0.324)\end{array}$ & $\begin{array}{c}0.877 \\
(0.328)\end{array}$ & $\begin{array}{c}0.893 \\
(0.310)\end{array}$ & $\begin{array}{c}0.879 \\
(0.326)\end{array}$ & $\begin{array}{c}0.891 \\
(0.312)\end{array}$ & $\begin{array}{c}0.868 \\
(0.338)\end{array}$ & $\begin{array}{c}0.894 \\
(0.308)\end{array}$ & $\begin{array}{c}0.831 \\
(0.375)\end{array}$ & $\begin{array}{c}0.851 \\
(0.357)\end{array}$ & $\begin{array}{c}0.817 \\
(0.387)\end{array}$ & $\begin{array}{c}0.877 \\
(0.328)\end{array}$ \\
\hline Married & No & No & No & No & No & No & No & No & No & No & No \\
\hline Children under 20 & No & No & No & No & No & No & No & No & No & No & No \\
\hline Children under 6 & No & No & No & No & No & No & No & No & No & No & No \\
\hline Household Income & $\begin{array}{c}548.943 \\
(373.616)\end{array}$ & $\begin{array}{c}499.281 \\
(357.399)\end{array}$ & $\begin{array}{c}470.869 \\
(336.370)\end{array}$ & $\begin{array}{c}465.917 \\
(324.055)\end{array}$ & $\begin{array}{c}456.821 \\
(329.592)\end{array}$ & $\begin{array}{c}457.463 \\
(332.213)\end{array}$ & $\begin{array}{c}459.801 \\
(328.239)\end{array}$ & $\begin{array}{c}425.819 \\
(304.129)\end{array}$ & $\begin{array}{c}458.463 \\
(295.607)\end{array}$ & $\begin{array}{c}428.966 \\
(305.892)\end{array}$ & $\begin{array}{c}497.250 \\
(350.350)\end{array}$ \\
\hline Personal Income & $\begin{array}{c}205.628 \\
(161.475)\end{array}$ & $\begin{array}{c}193.155 \\
(168.858)\end{array}$ & $\begin{array}{c}188.501 \\
(187.336)\end{array}$ & $\begin{array}{c}177.710 \\
(188.868)\end{array}$ & $\begin{array}{c}166.618 \\
(182.674)\end{array}$ & $\begin{array}{c}156.818 \\
(188.751)\end{array}$ & $\begin{array}{c}155.191 \\
(202.735)\end{array}$ & $\begin{array}{c}123.174 \\
(164.363)\end{array}$ & $\begin{array}{c}134.521 \\
(177.564)\end{array}$ & $\begin{array}{c}101.034 \\
(175.583)\end{array}$ & $\begin{array}{c}181.847 \\
(176.960)\end{array}$ \\
\hline Number of Adults & $\begin{array}{r}2.679 \\
(1.321) \\
\end{array}$ & $\begin{array}{c}2.490 \\
(1.308) \\
\end{array}$ & $\begin{array}{c}2.467 \\
(1.261) \\
\end{array}$ & $\begin{array}{c}2.442 \\
(1.213) \\
\end{array}$ & $\begin{array}{c}2.488 \\
(1.268) \\
\end{array}$ & $\begin{array}{c}2.488 \\
(1.153) \\
\end{array}$ & $\begin{array}{c}2.505 \\
(1.234) \\
\end{array}$ & $\begin{array}{c}2.589 \\
(1.155) \\
\end{array}$ & $\begin{array}{c}2.655 \\
(1.226) \\
\end{array}$ & $\begin{array}{c}2.655 \\
(1.045) \\
\end{array}$ & $\begin{array}{c}2.569 \\
(1.270) \\
\end{array}$ \\
\hline
\end{tabular}

Standard errors in parentheses $* * * \mathrm{p}<0.01, * * \mathrm{p}<0.05, * \mathrm{p}<0.1$ 
Table 1b Descriptive statistics, 20 to 60-year-old non-married women, Japan 2016

\begin{tabular}{|c|c|c|c|c|c|c|c|c|c|c|c|}
\hline & $\begin{array}{l}\text { Women in } \\
2016 \\
1 \\
(0-10 \% \text { of } \\
\text { Housewor } \\
\text { k) }\end{array}$ & $\begin{array}{c}2 \\
(10-20 \% \\
\text { of } \\
\text { Housewor } \\
\text { k) } \\
\end{array}$ & $\begin{array}{c}3 \\
(20-30 \% \\
\text { of } \\
\text { Housewor } \\
\text { k) } \\
\end{array}$ & $\begin{array}{c}4 \\
(30-40 \% \\
\text { of } \\
\text { Housewor } \\
\text { k) } \\
\end{array}$ & $\begin{array}{c}5 \\
(40-50 \% \\
\text { of } \\
\text { Housewor } \\
\text { k) } \\
\end{array}$ & $\begin{array}{c}6 \\
(50-60 \% \\
\text { of } \\
\text { Housewor } \\
\text { k) }\end{array}$ & $\begin{array}{c}7 \\
(60-70 \% \\
\text { of } \\
\text { Housewor } \\
\text { k) } \\
\end{array}$ & $\begin{array}{c}8 \\
(70-80 \% \\
\text { of } \\
\text { Housewor } \\
\text { k) } \\
\end{array}$ & $\begin{array}{c}9 \\
(80-90 \% \\
\text { of } \\
\text { Housewor } \\
\text { k) }\end{array}$ & $\begin{array}{c}10 \\
(90-100 \% \\
\text { of } \\
\text { Housewor } \\
\text { k) }\end{array}$ & Total \\
\hline Housework & $\begin{array}{c}39.914 \\
(16.395)\end{array}$ & $\begin{array}{l}83.359 \\
(7.453)\end{array}$ & $\begin{array}{l}113.674 \\
(7.411)\end{array}$ & $\begin{array}{l}142.735 \\
(7.501)\end{array}$ & $\begin{array}{l}173.641 \\
(7.418)\end{array}$ & $\begin{array}{l}209.118 \\
(11.506)\end{array}$ & $\begin{array}{l}252.864 \\
(12.392)\end{array}$ & $\begin{array}{l}297.463 \\
(11.020)\end{array}$ & $\begin{array}{l}360.338 \\
(25.108)\end{array}$ & $\begin{array}{c}514.278 \\
(102.031)\end{array}$ & $\begin{array}{c}133.027 \\
(111.267)\end{array}$ \\
\hline Education & $\begin{array}{l}13.340 \\
(2.337)\end{array}$ & $\begin{array}{l}13.227 \\
(2.324)\end{array}$ & $\begin{array}{l}13.094 \\
(2.225)\end{array}$ & $\begin{array}{l}13.105 \\
(2.231)\end{array}$ & $\begin{array}{l}12.985 \\
(2.497)\end{array}$ & $\begin{array}{l}13.028 \\
(2.277)\end{array}$ & $\begin{array}{l}12.889 \\
(2.273)\end{array}$ & $\begin{array}{l}13.012 \\
(1.993)\end{array}$ & $\begin{array}{l}13.062 \\
(2.294)\end{array}$ & $\begin{array}{l}12.680 \\
(2.487)\end{array}$ & $\begin{array}{l}13.158 \\
(2.313)\end{array}$ \\
\hline Paid Work Time & $\begin{array}{c}271.414 \\
(258.377)\end{array}$ & $\begin{array}{c}209.745 \\
(245.243)\end{array}$ & $\begin{array}{c}169.317 \\
(230.323)\end{array}$ & $\begin{array}{c}149.096 \\
(217.269)\end{array}$ & $\begin{array}{c}135.177 \\
(210.995)\end{array}$ & $\begin{array}{c}83.950 \\
(174.421)\end{array}$ & $\begin{array}{c}72.479 \\
(158.428)\end{array}$ & $\begin{array}{c}60.846 \\
(139.858)\end{array}$ & $\begin{array}{c}23.408 \\
(80.940)\end{array}$ & $\begin{array}{c}16.215 \\
(68.600)\end{array}$ & $\begin{array}{c}180.688 \\
(238.484)\end{array}$ \\
\hline Age & $\begin{array}{c}39.432 \\
(11.296)\end{array}$ & $\begin{array}{c}42.491 \\
(11.248)\end{array}$ & $\begin{array}{c}43.533 \\
(11.367)\end{array}$ & $\begin{array}{c}45.058 \\
(10.780)\end{array}$ & $\begin{array}{c}45.465 \\
(10.271)\end{array}$ & $\begin{array}{c}45.244 \\
(10.683)\end{array}$ & $\begin{array}{c}46.350 \\
(10.073)\end{array}$ & $\begin{array}{c}47.107 \\
(10.290)\end{array}$ & $\begin{array}{l}47.639 \\
(9.683)\end{array}$ & $\begin{array}{l}48.577 \\
(8.865)\end{array}$ & $\begin{array}{c}42.945 \\
(11.297)\end{array}$ \\
\hline Household size & $\begin{array}{c}2.715 \\
(1.387)\end{array}$ & $\begin{array}{c}2.542 \\
(1.355)\end{array}$ & $\begin{array}{c}2.518 \\
(1.355)\end{array}$ & $\begin{array}{c}2.479 \\
(1.326)\end{array}$ & $\begin{array}{c}2.436 \\
(1.272)\end{array}$ & $\begin{array}{c}2.590 \\
(1.294)\end{array}$ & $\begin{array}{c}2.648 \\
(1.432)\end{array}$ & $\begin{array}{c}2.668 \\
(1.171)\end{array}$ & $\begin{array}{c}2.642 \\
(1.159)\end{array}$ & $\begin{array}{c}2.870 \\
(1.483)\end{array}$ & $\begin{array}{c}2.614 \\
(1.353)\end{array}$ \\
\hline Employed & $\begin{array}{c}0.825 \\
(0.380)\end{array}$ & $\begin{array}{c}0.772 \\
(0.420)\end{array}$ & $\begin{array}{c}0.710 \\
(0.454)\end{array}$ & $\begin{array}{c}0.657 \\
(0.475)\end{array}$ & $\begin{array}{c}0.620 \\
(0.486)\end{array}$ & $\begin{array}{c}0.577 \\
(0.494)\end{array}$ & $\begin{array}{c}0.581 \\
(0.494)\end{array}$ & $\begin{array}{c}0.469 \\
(0.500)\end{array}$ & $\begin{array}{c}0.445 \\
(0.498)\end{array}$ & $\begin{array}{c}0.447 \\
(0.498)\end{array}$ & $\begin{array}{c}0.705 \\
(0.456)\end{array}$ \\
\hline Urban & $\begin{array}{c}0.888 \\
(0.315)\end{array}$ & $\begin{array}{c}0.894 \\
(0.308)\end{array}$ & $\begin{array}{c}0.878 \\
(0.327)\end{array}$ & $\begin{array}{c}0.889 \\
(0.315)\end{array}$ & $\begin{array}{c}0.888 \\
(0.316)\end{array}$ & $\begin{array}{c}0.882 \\
(0.322)\end{array}$ & $\begin{array}{c}0.906 \\
(0.293)\end{array}$ & $\begin{array}{c}0.905 \\
(0.294)\end{array}$ & $\begin{array}{c}0.907 \\
(0.291)\end{array}$ & $\begin{array}{c}0.891 \\
(0.312)\end{array}$ & $\begin{array}{c}0.890 \\
(0.313)\end{array}$ \\
\hline Married & No & No & No & No & No & No & No & No & No & No & No \\
\hline Children under 20 & No & No & No & No & No & No & No & No & No & No & No \\
\hline Children under 6 & No & No & No & No & No & No & No & No & No & No & No \\
\hline Household Income & $\begin{array}{c}553.695 \\
(372.917)\end{array}$ & $\begin{array}{c}502.328 \\
(355.613)\end{array}$ & $\begin{array}{c}472.696 \\
(327.447)\end{array}$ & $\begin{array}{c}448.494 \\
(315.875)\end{array}$ & $\begin{array}{c}446.750 \\
(302.634)\end{array}$ & $\begin{array}{c}468.908 \\
(319.613)\end{array}$ & $\begin{array}{c}481.732 \\
(345.822)\end{array}$ & $\begin{array}{c}437.537 \\
(306.418)\end{array}$ & $\begin{array}{c}435.634 \\
(309.167)\end{array}$ & $\begin{array}{c}436.268 \\
(368.631)\end{array}$ & $\begin{array}{c}497.737 \\
(348.567)\end{array}$ \\
\hline Personal Income & $\begin{array}{c}225.259 \\
(166.500)\end{array}$ & $\begin{array}{c}216.260 \\
(178.711)\end{array}$ & $\begin{array}{c}200.391 \\
(179.260)\end{array}$ & $\begin{array}{c}182.822 \\
(175.772)\end{array}$ & $\begin{array}{c}184.749 \\
(185.393)\end{array}$ & $\begin{array}{c}170.588 \\
(179.800)\end{array}$ & $\begin{array}{c}183.705 \\
(209.207)\end{array}$ & $\begin{array}{c}137.018 \\
(164.503)\end{array}$ & $\begin{array}{c}137.817 \\
(165.707)\end{array}$ & $\begin{array}{c}144.982 \\
(208.284)\end{array}$ & $\begin{array}{c}199.312 \\
(178.963)\end{array}$ \\
\hline Number of Adults & $\begin{array}{c}2.593 \\
(1.261) \\
\end{array}$ & $\begin{array}{c}2.418 \\
(1.215) \\
\end{array}$ & $\begin{array}{c}2.371 \\
(1.207) \\
\end{array}$ & $\begin{array}{c}2.334 \\
(1.139) \\
\end{array}$ & $\begin{array}{c}2.292 \\
(1.109) \\
\end{array}$ & $\begin{array}{c}2.458 \\
(1.161) \\
\end{array}$ & $\begin{array}{c}2.461 \\
(1.176) \\
\end{array}$ & $\begin{array}{c}2.496 \\
(1.064) \\
\end{array}$ & $\begin{array}{c}2.501 \\
(1.064) \\
\end{array}$ & $\begin{array}{c}2.634 \\
(1.302) \\
\end{array}$ & $\begin{array}{c}2.475 \\
(1.207) \\
\end{array}$ \\
\hline
\end{tabular}

Standard errors in parentheses $* * * \mathrm{p}<0.01, * * \mathrm{p}<0.05, * \mathrm{p}<0.1$ 
Table 2a Descriptive statistics, 20 to 60-year-old married women without children, Japan 2011.

\begin{tabular}{|c|c|c|c|c|c|c|c|c|c|c|c|}
\hline & $\begin{array}{l}\text { Women in } \\
2011 \\
1 \\
(0-10 \% \text { of } \\
\text { Housewor } \\
\text { k) }\end{array}$ & $\begin{array}{c}2 \\
(10-20 \% \\
\text { of } \\
\text { Housewor } \\
\mathrm{k}) \\
\end{array}$ & $\begin{array}{c}3 \\
(20-30 \% \\
\text { of } \\
\text { Housewor } \\
\text { k) }\end{array}$ & $\begin{array}{c}4 \\
(30-40 \% \\
\text { of } \\
\text { Housewor } \\
\text { k) }\end{array}$ & $\begin{array}{c}5 \\
(40-50 \% \\
\text { of } \\
\text { Housewor } \\
\text { k) } \\
\end{array}$ & $\begin{array}{c}6 \\
(50-60 \% \\
\text { of } \\
\text { Housewor } \\
\text { k) } \\
\end{array}$ & $\begin{array}{c}7 \\
(60-70 \% \\
\text { of } \\
\text { Housewor } \\
\text { k) } \\
\end{array}$ & $\begin{array}{c}8 \\
(70-80 \% \\
\text { of } \\
\text { Housewor } \\
\text { k) } \\
\end{array}$ & $\begin{array}{c}9 \\
(80-90 \% \\
\text { of } \\
\text { Housewor } \\
\text { k) } \\
\end{array}$ & $\begin{array}{c}10 \\
(90-100 \% \\
\text { of } \\
\text { Housewor } \\
\text { k) }\end{array}$ & Total \\
\hline Housework & $\begin{array}{c}44.549 \\
(15.111)\end{array}$ & $\begin{array}{l}83.723 \\
(7.401)\end{array}$ & $\begin{array}{c}113.644 \\
(7.414)\end{array}$ & $\begin{array}{l}143.169 \\
(7.472)\end{array}$ & $\begin{array}{l}173.088 \\
(7.479)\end{array}$ & $\begin{array}{l}209.410 \\
(12.122)\end{array}$ & $\begin{array}{l}254.234 \\
(12.364)\end{array}$ & $\begin{array}{l}299.108 \\
(11.817)\end{array}$ & $\begin{array}{l}361.732 \\
(25.108)\end{array}$ & $\begin{array}{l}509.464 \\
(91.863)\end{array}$ & $\begin{array}{r}218.020 \\
(133.514)\end{array}$ \\
\hline Education & $\begin{array}{l}12.835 \\
(1.858)\end{array}$ & $\begin{array}{l}12.751 \\
(1.851)\end{array}$ & $\begin{array}{l}12.709 \\
(1.819)\end{array}$ & $\begin{array}{l}12.657 \\
(1.729)\end{array}$ & $\begin{array}{l}12.607 \\
(1.741)\end{array}$ & $\begin{array}{l}12.689 \\
(1.702)\end{array}$ & $\begin{array}{l}12.626 \\
(1.656)\end{array}$ & $\begin{array}{l}12.698 \\
(1.748)\end{array}$ & $\begin{array}{l}12.729 \\
(1.753)\end{array}$ & $\begin{array}{l}12.688 \\
(1.756)\end{array}$ & $\begin{array}{l}12.699 \\
(1.760)\end{array}$ \\
\hline Paid Work Time & $\begin{array}{c}301.109 \\
(261.145)\end{array}$ & $\begin{array}{c}273.028 \\
(247.293)\end{array}$ & $\begin{array}{c}256.855 \\
(240.811)\end{array}$ & $\begin{array}{c}218.252 \\
(226.545)\end{array}$ & $\begin{array}{c}192.304 \\
(217.440)\end{array}$ & $\begin{array}{c}147.158 \\
(198.406)\end{array}$ & $\begin{array}{c}104.140 \\
(172.392)\end{array}$ & $\begin{array}{c}68.982 \\
(139.469)\end{array}$ & $\begin{array}{c}41.387 \\
(107.592)\end{array}$ & $\begin{array}{c}19.958 \\
(73.214)\end{array}$ & $\begin{array}{c}161.589 \\
(219.054)\end{array}$ \\
\hline Age & $\begin{array}{c}47.259 \\
(10.425)\end{array}$ & $\begin{array}{l}49.388 \\
(9.319)\end{array}$ & $\begin{array}{l}50.094 \\
(8.695)\end{array}$ & $\begin{array}{l}50.535 \\
(8.471)\end{array}$ & $\begin{array}{l}51.106 \\
(7.847)\end{array}$ & $\begin{array}{l}51.486 \\
(7.695)\end{array}$ & $\begin{array}{l}51.803 \\
(7.429)\end{array}$ & $\begin{array}{l}52.402 \\
(6.780)\end{array}$ & $\begin{array}{l}52.577 \\
(6.750)\end{array}$ & $\begin{array}{l}52.906 \\
(6.517)\end{array}$ & $\begin{array}{l}50.958 \\
(8.238)\end{array}$ \\
\hline Household size & $\begin{array}{c}2.835 \\
(1.316)\end{array}$ & $\begin{array}{c}2.893 \\
(1.291)\end{array}$ & $\begin{array}{c}3.014 \\
(1.333)\end{array}$ & $\begin{array}{c}3.000 \\
(1.267)\end{array}$ & $\begin{array}{c}3.037 \\
(1.306)\end{array}$ & $\begin{array}{c}3.097 \\
(1.311)\end{array}$ & $\begin{array}{c}3.108 \\
(1.298)\end{array}$ & $\begin{array}{c}3.176 \\
(1.278)\end{array}$ & $\begin{array}{c}3.214 \\
(1.305)\end{array}$ & $\begin{array}{c}3.329 \\
(1.286)\end{array}$ & $\begin{array}{c}3.070 \\
(1.307)\end{array}$ \\
\hline Employed & $\begin{array}{c}0.653 \\
(0.476)\end{array}$ & $\begin{array}{c}0.593 \\
(0.491)\end{array}$ & $\begin{array}{c}0.562 \\
(0.496)\end{array}$ & $\begin{array}{c}0.506 \\
(0.500)\end{array}$ & $\begin{array}{c}0.450 \\
(0.498)\end{array}$ & $\begin{array}{c}0.376 \\
(0.484)\end{array}$ & $\begin{array}{c}0.324 \\
(0.468)\end{array}$ & $\begin{array}{c}0.289 \\
(0.453)\end{array}$ & $\begin{array}{c}0.257 \\
(0.437)\end{array}$ & $\begin{array}{c}0.243 \\
(0.429)\end{array}$ & $\begin{array}{c}0.423 \\
(0.494)\end{array}$ \\
\hline Urban & $\begin{array}{c}0.852 \\
(0.355)\end{array}$ & $\begin{array}{c}0.853 \\
(0.355)\end{array}$ & $\begin{array}{c}0.855 \\
(0.352)\end{array}$ & $\begin{array}{c}0.845 \\
(0.362)\end{array}$ & $\begin{array}{c}0.858 \\
(0.349)\end{array}$ & $\begin{array}{c}0.862 \\
(0.345)\end{array}$ & $\begin{array}{c}0.863 \\
(0.344)\end{array}$ & $\begin{array}{c}0.877 \\
(0.329)\end{array}$ & $\begin{array}{c}0.867 \\
(0.340)\end{array}$ & $\begin{array}{c}0.847 \\
(0.360)\end{array}$ & $\begin{array}{c}0.858 \\
(0.349)\end{array}$ \\
\hline Married & Yes & Yes & Yes & Yes & Yes & Yes & Yes & Yes & Yes & Yes & Yes \\
\hline Children under 20 & No & No & No & No & No & No & No & No & No & No & No \\
\hline Children under 6 & No & No & No & No & No & No & No & No & No & No & No \\
\hline Household Income & $\begin{array}{c}706.760 \\
(392.904)\end{array}$ & $\begin{array}{c}722.913 \\
(407.900)\end{array}$ & $\begin{array}{c}734.726 \\
(402.326)\end{array}$ & $\begin{array}{c}722.642 \\
(392.226)\end{array}$ & $\begin{array}{c}724.717 \\
(397.734)\end{array}$ & $\begin{array}{c}723.199 \\
(398.706)\end{array}$ & $\begin{array}{c}729.768 \\
(401.740)\end{array}$ & $\begin{array}{c}742.773 \\
(410.549)\end{array}$ & $\begin{array}{c}740.946 \\
(403.628)\end{array}$ & $\begin{array}{c}746.565 \\
(408.292)\end{array}$ & $\begin{array}{r}729.029 \\
(401.327)\end{array}$ \\
\hline Personal Income & $\begin{array}{c}205.614 \\
(201.922)\end{array}$ & $\begin{array}{c}189.514 \\
(206.429)\end{array}$ & $\begin{array}{c}171.302 \\
(184.425)\end{array}$ & $\begin{array}{c}153.671 \\
(183.154)\end{array}$ & $\begin{array}{c}137.474 \\
(174.126)\end{array}$ & $\begin{array}{c}121.461 \\
(158.598)\end{array}$ & $\begin{array}{c}105.595 \\
(160.258)\end{array}$ & $\begin{array}{c}95.747 \\
(147.382)\end{array}$ & $\begin{array}{c}89.021 \\
(155.592)\end{array}$ & $\begin{array}{c}84.390 \\
(149.875)\end{array}$ & $\begin{array}{r}134.750 \\
(177.445)\end{array}$ \\
\hline Number of Adults & $\begin{array}{c}2.693 \\
(1.079) \\
\end{array}$ & $\begin{array}{c}2.766 \\
(1.088) \\
\end{array}$ & $\begin{array}{c}2.866 \\
(1.123) \\
\end{array}$ & $\begin{array}{c}2.870 \\
(1.076) \\
\end{array}$ & $\begin{array}{c}2.911 \\
(1.111) \\
\end{array}$ & $\begin{array}{c}2.957 \\
(1.128) \\
\end{array}$ & $\begin{array}{c}2.974 \\
(1.122) \\
\end{array}$ & $\begin{array}{c}3.043 \\
(1.117) \\
\end{array}$ & $\begin{array}{c}3.084 \\
(1.105) \\
\end{array}$ & $\begin{array}{c}3.180 \\
(1.122) \\
\end{array}$ & $\begin{array}{c}2.934 \\
(1.116) \\
\end{array}$ \\
\hline$N$ & 24543 & & & & & & & & & & \\
\hline
\end{tabular}

Standard errors in parentheses $* * * \mathrm{p}<0.01, * * \mathrm{p}<0.05, * \mathrm{p}<0.1$ 
Table 2b Descriptive statistics, 20 to 60-year-old married women without children, Japan 2016

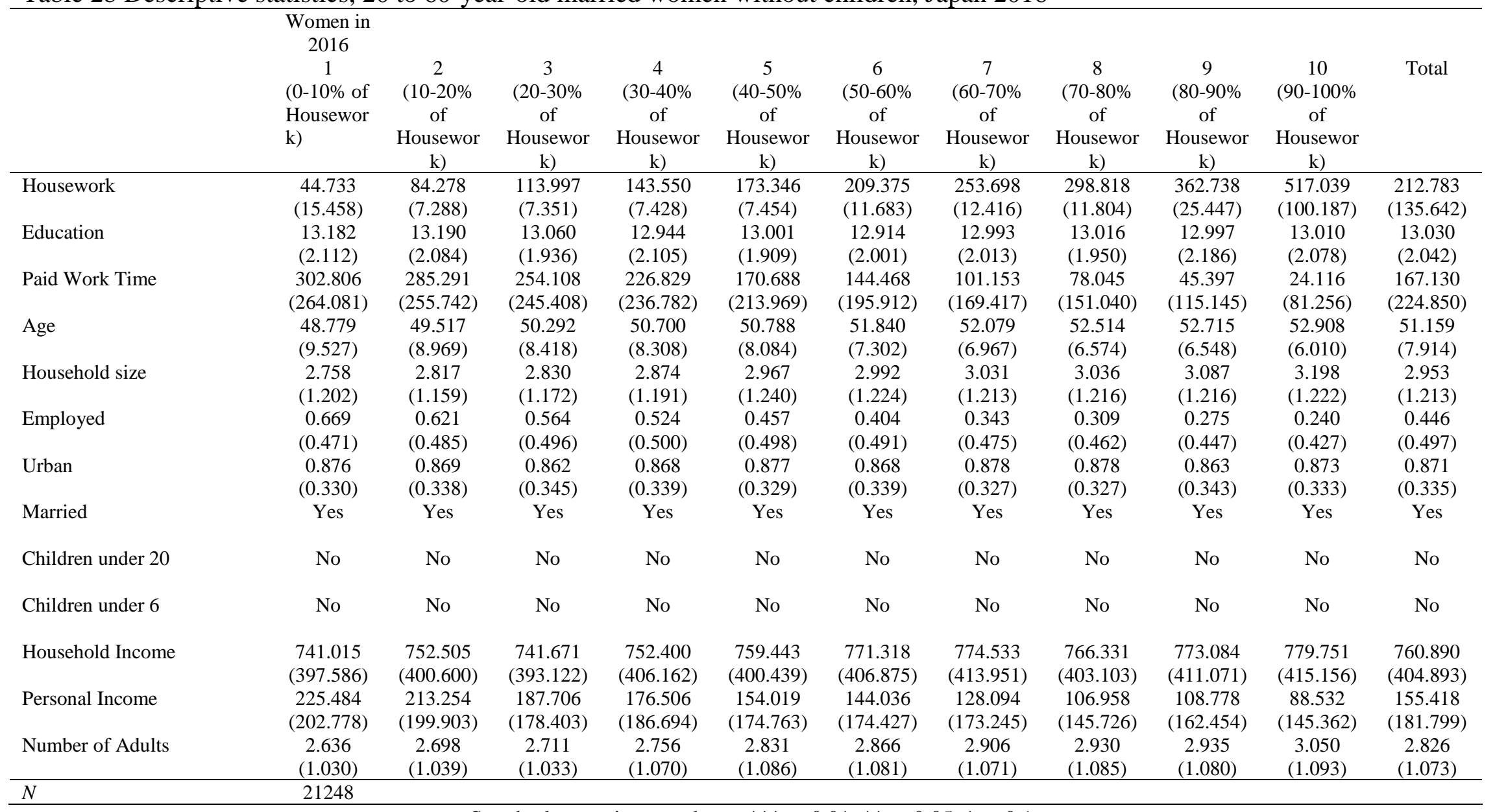

Standard errors in parentheses $* * * \mathrm{p}<0.01, * * \mathrm{p}<0.05, * \mathrm{p}<0.1$ 
Table 3a Descriptive statistics, 20 to 60-year-old married women with children, Japan 2011

\begin{tabular}{|c|c|c|c|c|c|c|c|c|c|c|c|}
\hline & $\begin{array}{l}\text { Women in } \\
2011 \\
1 \\
(0-10 \% \text { of } \\
\text { Housewor } \\
\text { k) }\end{array}$ & $\begin{array}{c}2 \\
(10-20 \% \\
\text { of } \\
\text { Housewor } \\
\text { k) }\end{array}$ & $\begin{array}{c}3 \\
(20-30 \% \\
\text { of } \\
\text { Housewor } \\
\text { k) }\end{array}$ & $\begin{array}{c}4 \\
(30-40 \% \\
\text { of } \\
\text { Housewor } \\
\text { k) }\end{array}$ & $\begin{array}{c}5 \\
(40-50 \% \\
\text { of } \\
\text { Housewor } \\
\text { k) }\end{array}$ & $\begin{array}{c}6 \\
(50-60 \% \\
\text { of } \\
\text { Housewor } \\
\text { k) }\end{array}$ & $\begin{array}{c}7 \\
(60-70 \% \\
\text { of } \\
\text { Housewor } \\
\text { k) }\end{array}$ & $\begin{array}{c}8 \\
(70-80 \% \\
\text { of } \\
\text { Housewor } \\
\text { k) }\end{array}$ & $\begin{array}{c}9 \\
(80-90 \% \\
\text { of } \\
\text { Housewor } \\
\text { k) }\end{array}$ & $\begin{array}{c}10 \\
(90-100 \% \\
\text { of } \\
\text { Housewor } \\
\text { k) }\end{array}$ & Total \\
\hline Housework & $\begin{array}{c}45.974 \\
(15.061)\end{array}$ & $\begin{array}{l}83.938 \\
(7.363)\end{array}$ & $\begin{array}{c}113.994 \\
(7.351)\end{array}$ & $\begin{array}{c}143.256 \\
(7.463)\end{array}$ & $\begin{array}{c}173.199 \\
(7.468)\end{array}$ & $\begin{array}{c}209.772 \\
(11.897)\end{array}$ & $\begin{array}{l}254.029 \\
(12.442)\end{array}$ & $\begin{array}{c}299.158 \\
(12.051)\end{array}$ & $\begin{array}{l}362.126 \\
(25.323)\end{array}$ & $\begin{array}{l}511.198 \\
(95.977)\end{array}$ & $\begin{array}{c}236.076 \\
(133.043)\end{array}$ \\
\hline Education & $\begin{array}{l}13.159 \\
(1.705)\end{array}$ & $\begin{array}{l}13.201 \\
(1.702)\end{array}$ & $\begin{array}{l}13.165 \\
(1.674)\end{array}$ & $\begin{array}{l}13.219 \\
(1.710)\end{array}$ & $\begin{array}{l}13.188 \\
(1.720)\end{array}$ & $\begin{array}{l}13.218 \\
(1.691)\end{array}$ & $\begin{array}{l}13.275 \\
(1.645)\end{array}$ & $\begin{array}{l}13.242 \\
(1.699)\end{array}$ & $\begin{array}{l}13.286 \\
(1.676)\end{array}$ & $\begin{array}{l}13.289 \\
(1.645)\end{array}$ & $\begin{array}{l}13.231 \\
(1.685)\end{array}$ \\
\hline Paid Work Time & $\begin{array}{c}246.206 \\
(258.641)\end{array}$ & $\begin{array}{c}227.076 \\
(247.487)\end{array}$ & $\begin{array}{c}199.453 \\
(234.033)\end{array}$ & $\begin{array}{c}182.253 \\
(228.316)\end{array}$ & $\begin{array}{c}157.552 \\
(210.972)\end{array}$ & $\begin{array}{c}124.143 \\
(191.002)\end{array}$ & $\begin{array}{c}89.794 \\
(162.960)\end{array}$ & $\begin{array}{c}72.470 \\
(145.273)\end{array}$ & $\begin{array}{c}52.824 \\
(123.079)\end{array}$ & $\begin{array}{c}28.078 \\
(88.672)\end{array}$ & $\begin{array}{c}126.239 \\
(200.708)\end{array}$ \\
\hline Age & $\begin{array}{l}38.332 \\
(7.314)\end{array}$ & $\begin{array}{l}38.715 \\
(7.282)\end{array}$ & $\begin{array}{l}39.326 \\
(7.284)\end{array}$ & $\begin{array}{l}39.064 \\
(7.103)\end{array}$ & $\begin{array}{l}39.447 \\
(7.041)\end{array}$ & $\begin{array}{l}39.785 \\
(6.984)\end{array}$ & $\begin{array}{l}40.379 \\
(6.696)\end{array}$ & $\begin{array}{l}40.695 \\
(6.667)\end{array}$ & $\begin{array}{l}41.709 \\
(6.651)\end{array}$ & $\begin{array}{l}42.858 \\
(6.334)\end{array}$ & $\begin{array}{l}40.190 \\
(7.019)\end{array}$ \\
\hline Household size & $\begin{array}{c}4.330 \\
(1.299)\end{array}$ & $\begin{array}{c}4.201 \\
(1.208)\end{array}$ & $\begin{array}{c}4.283 \\
(1.221)\end{array}$ & $\begin{array}{c}4.257 \\
(1.195)\end{array}$ & $\begin{array}{c}4.217 \\
(1.152)\end{array}$ & $\begin{array}{c}4.234 \\
(1.139)\end{array}$ & $\begin{array}{c}4.251 \\
(1.115)\end{array}$ & $\begin{array}{c}4.301 \\
(1.233)\end{array}$ & $\begin{array}{c}4.295 \\
(1.182)\end{array}$ & $\begin{array}{c}4.403 \\
(1.148)\end{array}$ & $\begin{array}{c}4.277 \\
(1.183)\end{array}$ \\
\hline Employed & $\begin{array}{c}0.533 \\
(0.499)\end{array}$ & $\begin{array}{c}0.502 \\
(0.500)\end{array}$ & $\begin{array}{c}0.466 \\
(0.499)\end{array}$ & $\begin{array}{c}0.411 \\
(0.492)\end{array}$ & $\begin{array}{c}0.385 \\
(0.487)\end{array}$ & $\begin{array}{c}0.311 \\
(0.463)\end{array}$ & $\begin{array}{c}0.285 \\
(0.452)\end{array}$ & $\begin{array}{c}0.248 \\
(0.432)\end{array}$ & $\begin{array}{c}0.224 \\
(0.417)\end{array}$ & $\begin{array}{c}0.187 \\
(0.390)\end{array}$ & $\begin{array}{c}0.336 \\
(0.472)\end{array}$ \\
\hline Urban & $\begin{array}{c}0.855 \\
(0.353)\end{array}$ & $\begin{array}{c}0.867 \\
(0.340)\end{array}$ & $\begin{array}{c}0.883 \\
(0.321)\end{array}$ & $\begin{array}{c}0.857 \\
(0.351)\end{array}$ & $\begin{array}{c}0.876 \\
(0.329)\end{array}$ & $\begin{array}{c}0.884 \\
(0.320)\end{array}$ & $\begin{array}{c}0.879 \\
(0.326)\end{array}$ & $\begin{array}{c}0.884 \\
(0.321)\end{array}$ & $\begin{array}{c}0.884 \\
(0.320)\end{array}$ & $\begin{array}{c}0.882 \\
(0.323)\end{array}$ & $\begin{array}{c}0.876 \\
(0.329)\end{array}$ \\
\hline Married & Yes & Yes & Yes & Yes & Yes & Yes & Yes & Yes & Yes & Yes & Yes \\
\hline Children under 20 & Yes & Yes & Yes & Yes & Yes & Yes & Yes & Yes & Yes & Yes & Yes \\
\hline Children under 6 & $\begin{array}{c}0.462 \\
(0.499)\end{array}$ & $\begin{array}{c}0.466 \\
(0.499)\end{array}$ & $\begin{array}{c}0.412 \\
(0.492)\end{array}$ & $\begin{array}{c}0.436 \\
(0.496)\end{array}$ & $\begin{array}{c}0.404 \\
(0.491)\end{array}$ & $\begin{array}{c}0.396 \\
(0.489)\end{array}$ & $\begin{array}{c}0.361 \\
(0.480)\end{array}$ & $\begin{array}{c}0.362 \\
(0.481)\end{array}$ & $\begin{array}{c}0.296 \\
(0.456)\end{array}$ & $\begin{array}{c}0.245 \\
(0.430)\end{array}$ & $\begin{array}{c}0.375 \\
(0.484)\end{array}$ \\
\hline Household Income & $\begin{array}{c}687.186 \\
(354.376)\end{array}$ & $\begin{array}{c}678.284 \\
(338.641)\end{array}$ & $\begin{array}{c}683.774 \\
(350.103)\end{array}$ & $\begin{array}{c}666.779 \\
(325.949)\end{array}$ & $\begin{array}{c}662.484 \\
(327.807)\end{array}$ & $\begin{array}{c}661.750 \\
(325.105)\end{array}$ & $\begin{array}{c}680.791 \\
(333.662)\end{array}$ & $\begin{array}{c}675.271 \\
(335.181)\end{array}$ & $\begin{array}{c}693.367 \\
(346.877)\end{array}$ & $\begin{array}{c}720.034 \\
(359.088)\end{array}$ & $\begin{array}{c}680.633 \\
(339.358)\end{array}$ \\
\hline Personal Income & $\begin{array}{c}172.639 \\
(181.134)\end{array}$ & $\begin{array}{c}167.589 \\
(185.502)\end{array}$ & $\begin{array}{c}155.525 \\
(170.014)\end{array}$ & $\begin{array}{c}138.566 \\
(164.847)\end{array}$ & $\begin{array}{c}126.244 \\
(154.084)\end{array}$ & $\begin{array}{c}106.673 \\
(145.579)\end{array}$ & $\begin{array}{c}99.471 \\
(145.217)\end{array}$ & $\begin{array}{c}88.450 \\
(137.704)\end{array}$ & $\begin{array}{c}85.463 \\
(136.549)\end{array}$ & $\begin{array}{c}73.966 \\
(132.721)\end{array}$ & $\begin{array}{c}115.529 \\
(156.196)\end{array}$ \\
\hline Number of Adults & $\begin{array}{c}2.621 \\
(1.043)\end{array}$ & $\begin{array}{c}2.488 \\
(0.964)\end{array}$ & $\begin{array}{c}2.534 \\
(0.961)\end{array}$ & $\begin{array}{c}2.468 \\
(0.935)\end{array}$ & $\begin{array}{c}2.423 \\
(0.900)\end{array}$ & $\begin{array}{c}2.396 \\
(0.866)\end{array}$ & $\begin{array}{c}2.396 \\
(0.856)\end{array}$ & $\begin{array}{c}2.431 \\
(0.865)\end{array}$ & $\begin{array}{c}2.405 \\
(0.849)\end{array}$ & $\begin{array}{c}2.456 \\
(0.877)\end{array}$ & $\begin{array}{c}2.450 \\
(0.904)\end{array}$ \\
\hline$N$ & 32400 & & & & & & & & & & \\
\hline
\end{tabular}

Standard errors in parentheses $* * * \mathrm{p}<0.01, * * \mathrm{p}<0.05, * \mathrm{p}<0.1$ 
Table 3b Descriptive statistics, 20 to 60-year-old married women with children, Japan 2016

\begin{tabular}{|c|c|c|c|c|c|c|c|c|c|c|c|}
\hline & $\begin{array}{l}\text { Women in } \\
2016 \\
1 \\
\text { (0-10\% of } \\
\text { Housewor } \\
\text { k) }\end{array}$ & $\begin{array}{c}2 \\
(10-20 \% \\
\text { of } \\
\text { Housewor } \\
\text { k) }\end{array}$ & $\begin{array}{c}3 \\
(20-30 \% \\
\text { of } \\
\text { Housewor } \\
\text { k) }\end{array}$ & $\begin{array}{c}4 \\
(30-40 \% \\
\text { of } \\
\text { Housewor } \\
\text { k) }\end{array}$ & $\begin{array}{c}5 \\
(40-50 \% \\
\text { of } \\
\text { Housewor } \\
\text { k) }\end{array}$ & $\begin{array}{c}6 \\
(50-60 \% \\
\text { of } \\
\text { Housewor } \\
\text { k) }\end{array}$ & $\begin{array}{c}7 \\
(60-70 \% \\
\text { of } \\
\text { Housewor } \\
\text { k) }\end{array}$ & $\begin{array}{c}8 \\
(70-80 \% \\
\text { of } \\
\text { Housewor } \\
\text { k) }\end{array}$ & $\begin{array}{c}9 \\
(80-90 \% \\
\text { of } \\
\text { Housewor } \\
\text { k) }\end{array}$ & $\begin{array}{c}10 \\
(90-100 \% \\
\text { of } \\
\text { Housewor } \\
\text { k) }\end{array}$ & Total \\
\hline Housework & $\begin{array}{c}45.476 \\
(15.128)\end{array}$ & $\begin{array}{l}84.383 \\
(7.261)\end{array}$ & $\begin{array}{l}114.083 \\
(7.332)\end{array}$ & $\begin{array}{l}143.657 \\
(7.412)\end{array}$ & $\begin{array}{l}173.286 \\
(7.460)\end{array}$ & $\begin{array}{l}209.510 \\
(11.910)\end{array}$ & $\begin{array}{c}254.235 \\
(12.479)\end{array}$ & $\begin{array}{c}299.658 \\
(11.767)\end{array}$ & $\begin{array}{l}362.181 \\
(24.767)\end{array}$ & $\begin{array}{c}519.773 \\
(107.805)\end{array}$ & $\begin{array}{c}233.533 \\
(139.801)\end{array}$ \\
\hline Education & $\begin{array}{l}13.459 \\
(2.231)\end{array}$ & $\begin{array}{l}13.404 \\
(2.150)\end{array}$ & $\begin{array}{l}13.428 \\
(2.127)\end{array}$ & $\begin{array}{l}13.377 \\
(2.191)\end{array}$ & $\begin{array}{l}13.476 \\
(2.024)\end{array}$ & $\begin{array}{l}13.470 \\
(2.097)\end{array}$ & $\begin{array}{l}13.409 \\
(2.097)\end{array}$ & $\begin{array}{l}13.367 \\
(2.174)\end{array}$ & $\begin{array}{l}13.447 \\
(2.106)\end{array}$ & $\begin{array}{l}13.378 \\
(2.029)\end{array}$ & $\begin{array}{l}13.424 \\
(2.118)\end{array}$ \\
\hline Paid Work Time & $\begin{array}{c}233.503 \\
(260.029)\end{array}$ & $\begin{array}{c}215.669 \\
(247.146)\end{array}$ & $\begin{array}{c}200.658 \\
(241.369)\end{array}$ & $\begin{array}{c}185.700 \\
(224.818)\end{array}$ & $\begin{array}{c}163.722 \\
(213.106)\end{array}$ & $\begin{array}{c}135.309 \\
(197.619)\end{array}$ & $\begin{array}{c}106.555 \\
(177.584)\end{array}$ & $\begin{array}{c}87.073 \\
(161.229)\end{array}$ & $\begin{array}{c}62.104 \\
(136.185)\end{array}$ & $\begin{array}{c}30.729 \\
(93.101)\end{array}$ & $\begin{array}{c}134.701 \\
(206.632)\end{array}$ \\
\hline Age & $\begin{array}{l}39.002 \\
(7.274)\end{array}$ & $\begin{array}{l}39.841 \\
(7.176)\end{array}$ & $\begin{array}{l}40.012 \\
(7.068)\end{array}$ & $\begin{array}{l}40.636 \\
(7.140)\end{array}$ & $\begin{array}{l}40.687 \\
(6.918)\end{array}$ & $\begin{array}{l}41.128 \\
(6.916)\end{array}$ & $\begin{array}{l}41.428 \\
(6.586)\end{array}$ & $\begin{array}{l}42.299 \\
(6.712)\end{array}$ & $\begin{array}{l}42.793 \\
(6.416)\end{array}$ & $\begin{array}{l}43.751 \\
(6.254)\end{array}$ & $\begin{array}{l}41.289 \\
(6.950)\end{array}$ \\
\hline Household size & $\begin{array}{c}4.225 \\
(1.224)\end{array}$ & $\begin{array}{c}4.209 \\
(1.204)\end{array}$ & $\begin{array}{c}4.167 \\
(1.155)\end{array}$ & $\begin{array}{c}4.165 \\
(1.116)\end{array}$ & $\begin{array}{c}4.133 \\
(1.052)\end{array}$ & $\begin{array}{c}4.177 \\
(1.095)\end{array}$ & $\begin{array}{c}4.203 \\
(1.076)\end{array}$ & $\begin{array}{c}4.220 \\
(1.087)\end{array}$ & $\begin{array}{c}4.265 \\
(1.132)\end{array}$ & $\begin{array}{c}4.375 \\
(1.099)\end{array}$ & $\begin{array}{c}4.216 \\
(1.121)\end{array}$ \\
\hline Employed & $\begin{array}{c}0.561 \\
(0.496)\end{array}$ & $\begin{array}{c}0.522 \\
(0.500)\end{array}$ & $\begin{array}{c}0.495 \\
(0.500)\end{array}$ & $\begin{array}{c}0.455 \\
(0.498)\end{array}$ & $\begin{array}{c}0.413 \\
(0.493)\end{array}$ & $\begin{array}{c}0.383 \\
(0.486)\end{array}$ & $\begin{array}{c}0.323 \\
(0.468)\end{array}$ & $\begin{array}{c}0.291 \\
(0.454)\end{array}$ & $\begin{array}{c}0.252 \\
(0.434)\end{array}$ & $\begin{array}{c}0.208 \\
(0.406)\end{array}$ & $\begin{array}{c}0.377 \\
(0.485)\end{array}$ \\
\hline Urban & $\begin{array}{c}0.894 \\
(0.308)\end{array}$ & $\begin{array}{c}0.876 \\
(0.329)\end{array}$ & $\begin{array}{c}0.885 \\
(0.319)\end{array}$ & $\begin{array}{c}0.875 \\
(0.331)\end{array}$ & $\begin{array}{c}0.883 \\
(0.322)\end{array}$ & $\begin{array}{c}0.873 \\
(0.333)\end{array}$ & $\begin{array}{c}0.888 \\
(0.315)\end{array}$ & $\begin{array}{c}0.880 \\
(0.325)\end{array}$ & $\begin{array}{c}0.897 \\
(0.303)\end{array}$ & $\begin{array}{c}0.893 \\
(0.310)\end{array}$ & $\begin{array}{c}0.885 \\
(0.319)\end{array}$ \\
\hline Married & Yes & Yes & Yes & Yes & Yes & Yes & Yes & Yes & Yes & Yes & Yes \\
\hline Children under 20 & Yes & Yes & Yes & Yes & Yes & Yes & Yes & Yes & Yes & Yes & Yes \\
\hline Children under 6 & $\begin{array}{c}0.478 \\
(0.500)\end{array}$ & $\begin{array}{c}0.444 \\
(0.497)\end{array}$ & $\begin{array}{c}0.423 \\
(0.494)\end{array}$ & $\begin{array}{c}0.369 \\
(0.483)\end{array}$ & $\begin{array}{c}0.386 \\
(0.487)\end{array}$ & $\begin{array}{c}0.357 \\
(0.479)\end{array}$ & $\begin{array}{c}0.341 \\
(0.474)\end{array}$ & $\begin{array}{c}0.298 \\
(0.457)\end{array}$ & $\begin{array}{c}0.280 \\
(0.449)\end{array}$ & $\begin{array}{c}0.222 \\
(0.416)\end{array}$ & $\begin{array}{c}0.353 \\
(0.478)\end{array}$ \\
\hline Household Income & $\begin{array}{c}717.904 \\
(334.280)\end{array}$ & $\begin{array}{c}716.967 \\
(343.789)\end{array}$ & $\begin{array}{c}709.631 \\
(334.092)\end{array}$ & $\begin{array}{c}709.977 \\
(334.396)\end{array}$ & $\begin{array}{c}708.743 \\
(335.349)\end{array}$ & $\begin{array}{c}703.349 \\
(326.761)\end{array}$ & $\begin{array}{c}704.727 \\
(330.575)\end{array}$ & $\begin{array}{c}710.523 \\
(337.182)\end{array}$ & $\begin{array}{c}723.314 \\
(342.614)\end{array}$ & $\begin{array}{c}734.470 \\
(357.757)\end{array}$ & $\begin{array}{c}713.767 \\
(337.557)\end{array}$ \\
\hline Personal Income & $\begin{array}{c}189.753 \\
(180.213)\end{array}$ & $\begin{array}{c}183.358 \\
(177.467)\end{array}$ & $\begin{array}{c}175.943 \\
(179.251)\end{array}$ & $\begin{array}{c}156.304 \\
(167.989)\end{array}$ & $\begin{array}{c}147.467 \\
(163.648)\end{array}$ & $\begin{array}{c}136.053 \\
(158.722)\end{array}$ & $\begin{array}{c}117.761 \\
(145.431)\end{array}$ & $\begin{array}{c}111.084 \\
(150.050)\end{array}$ & $\begin{array}{c}102.321 \\
(145.790)\end{array}$ & $\begin{array}{c}89.747 \\
(141.965)\end{array}$ & $\begin{array}{r}136.976 \\
(163.106)\end{array}$ \\
\hline Number of Adults & $\begin{array}{c}2.462 \\
(0.903) \\
\end{array}$ & $\begin{array}{c}2.431 \\
(0.878) \\
\end{array}$ & $\begin{array}{c}2.375 \\
(0.843) \\
\end{array}$ & $\begin{array}{c}2.387 \\
(0.837) \\
\end{array}$ & $\begin{array}{c}2.313 \\
(0.757) \\
\end{array}$ & $\begin{array}{c}2.348 \\
(0.811)\end{array}$ & $\begin{array}{c}2.320 \\
(0.779)\end{array}$ & $\begin{array}{c}2.341 \\
(0.792)\end{array}$ & $\begin{array}{c}2.362 \\
(0.826)\end{array}$ & $\begin{array}{c}2.407 \\
(0.811)\end{array}$ & $\begin{array}{c}2.370 \\
(0.822)\end{array}$ \\
\hline
\end{tabular}

Standard errors in parentheses $* * * \mathrm{p}<0.01, * * \mathrm{p}<0.05, * \mathrm{p}<0.1$ 


\section{Independent and Control Variables}

The main independent variable - education - is measured in years spent in school. If to analyze the education variable across deciles of housework participation in Tables 1a-3b, we can see that there are few differences in the sample of all women. On average, women have about 13 years of education (a little more than high school).

Control variables include household and personal incomes, which are both measured in thousands of Japanese yen. The controls also include work time (measured in minutes on the diary day) and employment status ( $1=$ 'employed full-time or part-time', $0=$ 'otherwise'). Dummy variables are used for measuring marital status $(1=$ married, $0=$ otherwise), whether the respondent owns the house, where he/she resides, and whether the respondent resides in an urban area. The models also include the number of adults in the household and year-fixed effects.

Additionally, we control for the presence of children, which are coded as 1 for the individuals who have children under 20. We also use age in years trying to capture the age effects. Since there are usually considerable differences in diaries depending on the day of the week when the diary was collected, we also control for whether the diary day was completed for a weekday or on the weekend (1=weekday, $0=$ Saturday or Sunday).

\section{Models}

To analyze the effects of gender, marital status, and education on participation in routine housework, we also employ unconditional quantile regression models (UQR) (Firpo et al., 2009). UQR has been recently used in many family studies, particularly in the research of motherhood premiums (Budig \& Hodges, 2014; England, Bearak, Budig, \& Hodges, 2016; Killewald \& Bearak, 2014). It is preferred to the conditional quantile regression, which is more prone to 
biased results (Killewald \& Bearak, 2014). For a more detailed explanation on UQR please refer to Firpo et al. (2009), Killewald and Bearak (2014), and Cooke and Hook (2018).

The results presented in this paper are robust to the exclusion of correlates of the resource-based frameworks (household and personal income, as well as paid work time).

\section{Results and Discussions}

Following the suggestion in Firpo et al. (2009), Budig and Hodges (2014), Killewald and Bearak (2014), England et al. (2016), we run unconditional quantile regression (UQR) models on participation in housework for non-married women without children, married women without children, and married women with children. The summaries of the UQR estimates are represented in Figure 1 and Table 4. Negative effects mean that the association between educational level and participation in housework is reverse (as expected): the higher the education level, the lower the housework participation. The stronger negative effects mean that educational level is effective in reducing the housework burden. Conversely, if the effects are positive, it means that higher levels of education are associated with higher levels of housework involvement.

Table 4 summarizes the results of the UQR models, which can be found in full in the Appendix A. One main conclusion that can be drawn immediately from the table is that the results are mostly significant only for married women. These are also confirmed in the full tables in the Appendix. Thus, the limitations of the present findings are that the findings mostly apply to married women without children, whereas there are more uncertainties about the findings in other groups of women.

Table 4 Summary of UQR results for Japanese Women Education's Association with Housework 


\begin{tabular}{|c|c|c|c|c|c|}
\hline & \multicolumn{5}{|c|}{ UQR } \\
\hline & $\begin{array}{c}2 \\
(10-20 \% \text { of } \\
\text { Housework) }\end{array}$ & $\begin{array}{c}3 \\
(20-30 \% \text { of } \\
\text { Housework) }\end{array}$ & $\begin{array}{c}5 \\
(40-50 \% \text { of } \\
\text { Housework) }\end{array}$ & $\begin{array}{c}8 \\
\text { (70-80\% of } \\
\text { Housework) }\end{array}$ & $\begin{array}{c}9 \\
\text { (80-90\% of } \\
\text { Housework) }\end{array}$ \\
\hline Non-Married Women & $\begin{array}{c}-0.003 * * * \\
(0.001)\end{array}$ & $\begin{array}{l}-0.002 \\
(0.003)\end{array}$ & $\begin{array}{c}-0.006 \\
(0.005)\end{array}$ & $\begin{array}{c}0.003 \\
(0.007)\end{array}$ & $\begin{array}{c}0.016 \\
(0.013)\end{array}$ \\
\hline Married Women without Children & $\begin{array}{c}-0.004^{*} \\
(0.002)\end{array}$ & $\begin{array}{l}-0.004 \\
(0.004)\end{array}$ & $\begin{array}{c}0.011 * * * \\
(0.004)\end{array}$ & $\begin{array}{c}0.028 * * * \\
(0.007)\end{array}$ & $\begin{array}{c}0.025^{* * * *} \\
(0.008)\end{array}$ \\
\hline Married Women with Children & $\begin{array}{c}-0.000 \\
(0.004)\end{array}$ & $\begin{array}{c}0.001 \\
(0.002)\end{array}$ & $\begin{array}{c}0.003 \\
(0.003)\end{array}$ & $\begin{array}{c}0.005 \\
(0.005)\end{array}$ & $\begin{array}{c}-0.001 \\
(0.006)\end{array}$ \\
\hline
\end{tabular}

Standard errors in parentheses $* * * \mathrm{p}<0.01, * * \mathrm{p}<0.05, * \mathrm{p}<0.1$

Figure 1 shows that among Japanese women, marriage penalizes the effects of education, and the association between educational level and housework participation reverses, especially on the higher deciles of housework participation among married women. The black arrows in Figure 1 indicate the (expected) negative association of educational level with housework participation. These arrows show the deciles where higher educational levels are associated with lower housework participation. On the other hand, red arrows indicate where the association is positive. These arrows are in the deciles where higher educational levels are associated with higher housework participation. The relative strength of the association on different quantiles of participation in housework is represented by the size of an arrow in Figure 1. The figure, thus, shows that the positive association is stronger among married women without children in higher deciles of housework participation. This might indicate that married women's role deviance (such as not having children) might result in more gender performance in housework participation in this group on Japanese women. Conversely, among non-married women in Japan, the association between educational level and housework participation generally follows the expectations of the resource-based frameworks and Hypothesis 1. Mostly only for non-married women in Japan, the educational level has positive effects on the reduction of housework burden, while it does not for married women. 


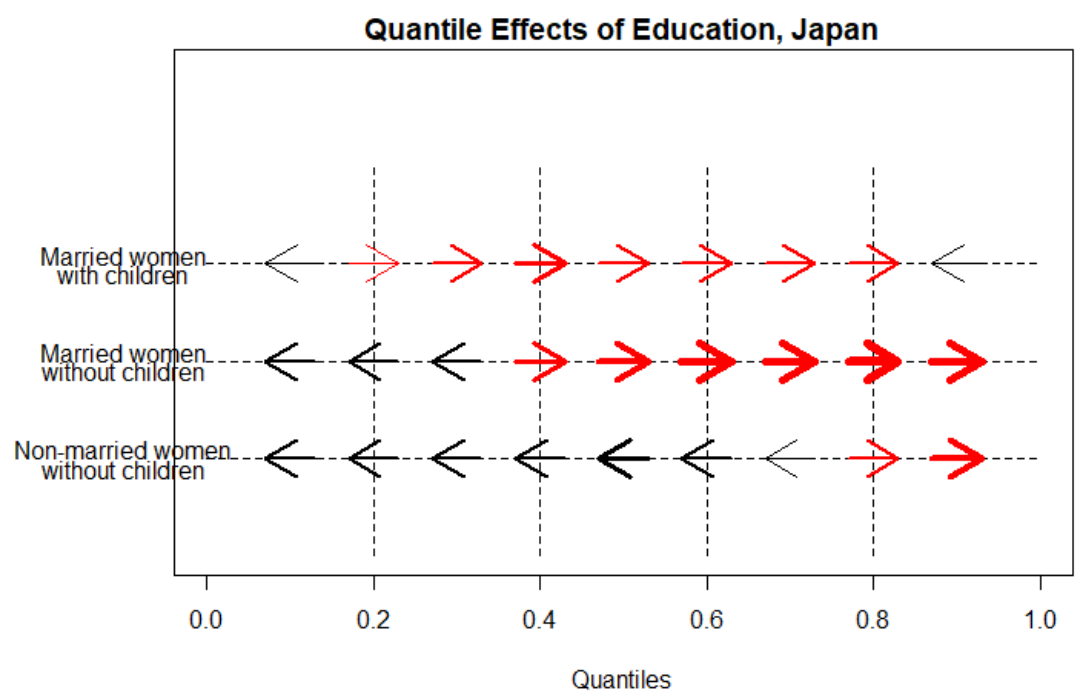

Figure 1 Quantile Effects of Education as Force Field

Education, therefore, helps to unburden only non-married women in Japan. However, it has an opposite effect on married women, especially those without children. It can be that until Japanese women are married, higher educated women do not feel the pressure of having to perform more housework. However, the pressure rises when they marry and continues to rise until they have kids. Married women without children are continuously under pressure to increase their housework performance, especially if they are highly educated. When they have kids, the pressure subsides but does not evaporate.

\section{Conclusions}

The resource-based approaches to housework participation might apply differently in different contexts and on different life-stages. The ways how these factors work in different cultural contexts are often underplayed (Kan \& Hertog, 2017; Kan \& Laurie, 2016). The results of the present study show that even though education provides non-married Japanese women with an ability to reduce their housework burden, married women with higher educational levels are unlikely to do so. We know from the studies in the global north that education is associated 
with the reduction in housework time among women (Kan \& Gershuny, 2010; Kan, Sullivan, \& Gershuny, 2011; Kolpashnikova, 2016, 2018). In other cultural contexts, such as that of the Japanese society analyzed in the present paper, we find the evidence that the association of education is more complex and culturally-bound than the researchers of housework assume. Thus, we find that married Japanese women, especially those without children, actually increase their housework participation with the increase in their educational level. It is likely that education as the socialization medium may convey different value sets in different countries, moreover, the institution of marriage might place expectations on women other than those expected in the global north.

The future research of gendered division of housework could benefit from developing theoretical frameworks which take into consideration these contextual nuances. Specifically, we urge the proponents of the resource-based theoretical stances to reconsider and take into account cultural and other social factors, not only economic, which are equally important when we analyze gender inequality. At times, the effects of sociocultural factors may be more meaningful than that of the resource-based explanations.

\section{References}

Baxter, J., Hewitt, B., \& Haynes, M. (2008). Life course transitions and housework: Marriage, parenthood, and time on housework. Journal of Marriage and Family, 70(2), 259-272.

Budig, M. J., \& Hodges, M. J. (2014). Statistical models and empirical evidence for differences in the motherhood penalty across the earnings distribution. American Sociological Review, 79(2), 358-364.

Cooke, L. P., \& Hook, J. L. (2018). Productivity or Gender? The Impact of Domestic Tasks Across the Wage Distribution. Journal of Marriage and Family, 80, 721-736. doi:10.1111/jomf.12467

England, P., Bearak, J., Budig, M. J., \& Hodges, M. J. (2016). Do highly paid, highly skilled women experience the largest motherhood penalty? American Sociological Review, 
81(6), 1161-1189.

England, P., Garcia-Beaulieu, C., \& Ross, M. (2004). Women's employment among blacks, whites, and three groups of Latinas: Do more privileged women have higher employment? Gender \& Society, 18(4), 494-509.

Firpo, S., Fortin, N. M., \& Lemieux, T. (2009). Unconditional quantile regressions. Econometrica, 77(3), 953-973.

Kan, M. Y., \& Gershuny, J. (2010). Gender segregation and bargaining in domestic labour: Evidence from longitudinal time-use data. In J. L. Scott, R. Crompton, \& C. Lyonette (Eds.), Gender inequalities in the 21 st century: New barriers and continuing constraints (pp. 153-173). Cheltenham, UK: Edward Elgar.

Kan, M. Y., \& Hertog, E. (2017). Domestic division of labour and fertility preference in China, Japan, South Korea, and Taiwan. Demographic Research, 36, 557-588. doi:10.4054/DemRes.2017.36.18

Kan, M. Y., \& Laurie, H. (2016). Gender, ethnicity and household labour in married and cohabiting couples in the UK. Institute for Social \& Economic Research. ISER Working Paper Series 2016-01.

Kan, M. Y., \& Pudney, S. (2008). Measurement Error in Stylized and Diary Data on Time Use. Sociological Methodology, 38(1), 101-132.

Kan, M. Y., Sullivan, O., \& Gershuny, J. (2011). Gender convergence in domestic work: Discerning the effects of interactional and institutional barriers from large-scale data. Sociology, 45(2), 234-251. doi:10.1177/0038038510394014

Killewald, A., \& Bearak, J. (2014). Is the motherhood penalty larger for low-wage women? A comment on quantile regression. American Sociological Review, 79(2), 350-357.

Kolpashnikova, K. (2016). Housework in Canada: uneven convergence of the gender gap in domestic tasks, 1986-2010. University of British Columbia, Vancouver, BC.

Kolpashnikova, K. (2018). American Househusbands: New Time Use Evidence of Gender Display, 2003-2016. Social Indicators Research, 140(3), 1259-1277. doi:10.1007/s11205-017-1813-z

Rubery, J., Smith, M., \& Fagan, C. (1999). Women's employment in Europe: Trends and prospects: Psychology Press.

South, S. J., \& Spitze, G. (1994). Housework in marital and nonmarital households. American Sociological Review, 327-347.

Zhou, M. (2017). Motherhood, Employment, and the Dynamics of Women's Gender Attitudes. Gender \& Society, 31(6), 751-776. 


\section{Appendix}

Table A1 UQR Coefficients, Japanese Non-Married Women without Children, STULA 2011 and 2016

\begin{tabular}{|c|c|c|c|c|c|c|c|c|c|}
\hline & $\begin{array}{c}1 \\
(0-10 \% \text { of } \\
\text { Housework) }\end{array}$ & $\begin{array}{c}2 \\
(10-20 \% \text { of } \\
\text { Housework) }\end{array}$ & $\begin{array}{c}3 \\
\text { (20-30\% of } \\
\text { Housework) } \\
\end{array}$ & $\begin{array}{c}4 \\
\text { (30-40\% of } \\
\text { Housework) } \\
\end{array}$ & $\begin{array}{c}5 \\
(40-50 \% \text { of } \\
\text { Housework) } \\
\end{array}$ & $\begin{array}{c}6 \\
(50-60 \% \text { of } \\
\text { Housework) } \\
\end{array}$ & $\begin{array}{c}7 \\
\text { (60-70\% of } \\
\text { Housework) } \\
\end{array}$ & $\begin{array}{c}8 \\
\text { (70-80\% of } \\
\text { Housework) }\end{array}$ & $\begin{array}{c}9 \\
\text { (80-90\% of } \\
\text { Housework) } \\
\end{array}$ \\
\hline Education & $\begin{array}{c}-0.00266 * * * \\
(0.000978)\end{array}$ & $\begin{array}{l}-0.00214 \\
(0.00267)\end{array}$ & $\begin{array}{c}-0.00365^{*} \\
(0.00194)\end{array}$ & $\begin{array}{l}-0.00403 \\
(0.00370)\end{array}$ & $\begin{array}{l}-0.00621 \\
(0.00498)\end{array}$ & $\begin{array}{l}-0.00469 \\
(0.00378)\end{array}$ & $\begin{array}{l}-0.000157 \\
(0.00797)\end{array}$ & $\begin{array}{c}0.00308 \\
(0.00746)\end{array}$ & $\begin{array}{c}0.0159 \\
(0.0130)\end{array}$ \\
\hline Paid Work & $\begin{array}{c}-0.000177 * * * \\
(1.21 \mathrm{e}-05)\end{array}$ & $\begin{array}{c}-0.000698 * * * \\
(2.84 \mathrm{e}-05)\end{array}$ & $\begin{array}{c}-0.000615 * * * \\
(1.99 \mathrm{e}-05)\end{array}$ & $\begin{array}{c}-0.00126 * * * \\
(3.64 \mathrm{e}-05)\end{array}$ & $\begin{array}{c}-0.00180 * * * \\
(4.61 \mathrm{e}-05)\end{array}$ & $\begin{array}{c}-0.00136 * * * \\
(3.47 \mathrm{e}-05)\end{array}$ & $\begin{array}{c}-0.00263 * * * \\
(6.78 \mathrm{e}-05)\end{array}$ & $\begin{array}{c}-0.00223 * * * \\
(6.09 \mathrm{e}-05)\end{array}$ & $\begin{array}{r}-0.00280 * * * \\
(0.000101)\end{array}$ \\
\hline Household Inc & $\begin{array}{l}0.00509 \\
(0.0102)\end{array}$ & $\begin{array}{l}-0.00497 \\
(0.0249)\end{array}$ & $\begin{array}{l}0.00271 \\
(0.0176)\end{array}$ & $\begin{array}{l}-0.0148 \\
(0.0328)\end{array}$ & $\begin{array}{l}-0.0371 \\
(0.0428)\end{array}$ & $\begin{array}{l}-0.0178 \\
(0.0323)\end{array}$ & $\begin{array}{c}0.0632 \\
(0.0665)\end{array}$ & $\begin{array}{c}0.0563 \\
(0.0618)\end{array}$ & $\begin{array}{r}-0.0420 \\
(0.109)\end{array}$ \\
\hline Personal Inc & $\begin{array}{l}-0.00990 \\
(0.0174)\end{array}$ & $\begin{array}{l}-0.0150 \\
(0.0440)\end{array}$ & $\begin{array}{l}-0.00587 \\
(0.0320)\end{array}$ & $\begin{array}{l}-0.00633 \\
(0.0611)\end{array}$ & $\begin{array}{c}0.0176 \\
(0.0825)\end{array}$ & $\begin{array}{l}-0.0192 \\
(0.0632)\end{array}$ & $\begin{array}{l}-0.0750 \\
(0.130)\end{array}$ & $\begin{array}{l}-0.0547 \\
(0.121)\end{array}$ & $\begin{array}{l}-0.324 \\
(0.201)\end{array}$ \\
\hline Weekday & $\begin{array}{l}-0.00401 \\
(0.00496)\end{array}$ & $\begin{array}{c}0.0113 \\
(0.0121)\end{array}$ & $\begin{array}{c}0.0108 \\
(0.00866)\end{array}$ & $\begin{array}{l}0.0342 * * \\
(0.0164)\end{array}$ & $\begin{array}{c}0.0445^{* *} \\
(0.0216)\end{array}$ & $\begin{array}{c}0.0201 \\
(0.0164)\end{array}$ & $\begin{array}{c}0.0248 \\
(0.0333)\end{array}$ & $\begin{array}{c}0.0290 \\
(0.0311)\end{array}$ & $\begin{array}{c}0.0610 \\
(0.0549)\end{array}$ \\
\hline Age & $\begin{array}{c}0.00310 * * * \\
(0.000202)\end{array}$ & $\begin{array}{l}0.0140 * * * \\
(0.000514)\end{array}$ & $\begin{array}{l}0.0110 * * * \\
(0.000370)\end{array}$ & $\begin{array}{l}0.0238 * * * \\
(0.000698)\end{array}$ & $\begin{array}{l}0.0328 * * * \\
(0.000936)\end{array}$ & $\begin{array}{l}0.0248 * * * \\
(0.000711)\end{array}$ & $\begin{array}{c}0.0490 * * * \\
(0.00148)\end{array}$ & $\begin{array}{c}0.0418 * * * \\
(0.00140)\end{array}$ & $\begin{array}{c}0.0556 * * * \\
(0.00254)\end{array}$ \\
\hline Household Size & $\begin{array}{c}0.0123 * * * \\
(0.00380)\end{array}$ & $\begin{array}{c}0.0361 * * * \\
(0.0104)\end{array}$ & $\begin{array}{c}0.0261 * * * \\
(0.00756)\end{array}$ & $\begin{array}{c}0.0698 * * * \\
(0.0143)\end{array}$ & $\begin{array}{l}0.130 * * * \\
(0.0194)\end{array}$ & $\begin{array}{l}0.104 * * * \\
(0.0150)\end{array}$ & $\begin{array}{c}0.158 * * * \\
(0.0327)\end{array}$ & $\begin{array}{c}0.139 * * * \\
(0.0308)\end{array}$ & $\begin{array}{c}0.165 * * * \\
(0.0546)\end{array}$ \\
\hline Owns Home & $\begin{array}{c}-0.0157 * * * \\
(0.00509)\end{array}$ & $\begin{array}{c}-0.0523 * * * \\
(0.0130)\end{array}$ & $\begin{array}{c}-0.0396 * * * \\
(0.00949)\end{array}$ & $\begin{array}{c}-0.0689 * * * \\
(0.0183)\end{array}$ & $\begin{array}{l}-0.0317 \\
(0.0248)\end{array}$ & $\begin{array}{l}-0.0189 \\
(0.0190)\end{array}$ & $\begin{array}{l}-0.0202 \\
(0.0396)\end{array}$ & $\begin{array}{l}0.00919 \\
(0.0370)\end{array}$ & $\begin{array}{c}0.0176 \\
(0.0645)\end{array}$ \\
\hline Employed & $\begin{array}{c}-0.0204 * * * \\
(0.00538)\end{array}$ & $\begin{array}{c}-0.0932 * * * \\
(0.0142)\end{array}$ & $\begin{array}{c}-0.0907 * * * \\
(0.0106)\end{array}$ & $\begin{array}{c}-0.238 * * * \\
(0.0208)\end{array}$ & $\begin{array}{c}-0.393 * * * \\
(0.0290)\end{array}$ & $\begin{array}{c}-0.310 * * * \\
(0.0224)\end{array}$ & $\begin{array}{c}-0.670 * * * \\
(0.0474)\end{array}$ & $\begin{array}{c}-0.604 * * * \\
(0.0449)\end{array}$ & $\begin{array}{c}-0.853 * * * \\
(0.0788)\end{array}$ \\
\hline Urban & $\begin{array}{c}-0.0196 * * * \\
(0.00618)\end{array}$ & $\begin{array}{l}-0.0164 \\
(0.0164)\end{array}$ & $\begin{array}{c}-0.00396 \\
(0.0118)\end{array}$ & $\begin{array}{l}-0.0248 \\
(0.0225)\end{array}$ & $\begin{array}{l}-0.0342 \\
(0.0296)\end{array}$ & $\begin{array}{l}-0.0362 \\
(0.0227)\end{array}$ & $\begin{array}{l}-0.0565 \\
(0.0468)\end{array}$ & $\begin{array}{l}-0.0795 * \\
(0.0447)\end{array}$ & $\begin{array}{c}-0.188 * * \\
(0.0806)\end{array}$ \\
\hline Year & $\begin{array}{l}-0.000607 \\
(0.000851)\end{array}$ & $\begin{array}{l}-0.00209 \\
(0.00212)\end{array}$ & $\begin{array}{l}0.000692 \\
(0.00153)\end{array}$ & $\begin{array}{l}0.000689 \\
(0.00291)\end{array}$ & $\begin{array}{c}-0.000163 \\
(0.00388)\end{array}$ & $\begin{array}{l}-2.82 \mathrm{e}-06 \\
(0.00295)\end{array}$ & $\begin{array}{l}-0.0115^{*} \\
(0.00612)\end{array}$ & $\begin{array}{l}-0.0109^{*} \\
(0.00574)\end{array}$ & $\begin{array}{c}-0.0324 * * * \\
(0.0100)\end{array}$ \\
\hline \# adults & $\begin{array}{c}-0.0217 * * * \\
(0.00460)\end{array}$ & $\begin{array}{c}-0.0498^{* * * *} \\
(0.0123)\end{array}$ & $\begin{array}{c}-0.0308 * * * \\
(0.00892)\end{array}$ & $\begin{array}{c}-0.0784 * * * \\
(0.0168)\end{array}$ & $\begin{array}{c}-0.130 * * * \\
(0.0227)\end{array}$ & $\begin{array}{c}-0.0985 * * * \\
(0.0175)\end{array}$ & $\begin{array}{c}-0.118 * * * \\
(0.0381)\end{array}$ & $\begin{array}{c}-0.0780 * * \\
(0.0358)\end{array}$ & $\begin{array}{l}-0.0330 \\
(0.0645)\end{array}$ \\
\hline Constant & $\begin{array}{c}31.28 * * * \\
(1.714)\end{array}$ & $\begin{array}{c}48.98 * * * \\
(4.262)\end{array}$ & $\begin{array}{c}58.47 * * * \\
(3.077)\end{array}$ & $\begin{array}{c}73.24 * * * \\
(5.864)\end{array}$ & $\begin{array}{c}104.7 * * * \\
(7.803)\end{array}$ & $\begin{array}{c}119.6^{* * *} \\
(5.935)\end{array}$ & $\begin{array}{c}186.9 * * * \\
(12.30)\end{array}$ & $\begin{array}{c}230.8 * * * \\
(11.56)\end{array}$ & $\begin{array}{c}348.7 * * * \\
(20.18)\end{array}$ \\
\hline $\begin{array}{l}\text { Observations } \\
\text { R-squared }\end{array}$ & $\begin{array}{c}20,243 \\
0.041\end{array}$ & $\begin{array}{c}20,243 \\
0.098\end{array}$ & $\begin{array}{c}20,243 \\
0.129\end{array}$ & $\begin{array}{c}20,243 \\
0.158\end{array}$ & $\begin{array}{c}20,243 \\
0.180\end{array}$ & $\begin{array}{c}20,243 \\
0.181\end{array}$ & $\begin{array}{c}20,243 \\
0.166\end{array}$ & $\begin{array}{c}20,243 \\
0.143\end{array}$ & $\begin{array}{c}20,243 \\
0.089\end{array}$ \\
\hline
\end{tabular}

Standard errors in parentheses $* * * p<0.01, * * p<0.05, * p<0.1$ 
Table A2 UQR Coefficients, Japanese Married Women without Children, STULA 2011 and 2016

\begin{tabular}{|c|c|c|c|c|c|c|c|c|c|}
\hline & $\begin{array}{c}1 \\
(0-10 \% \text { of } \\
\text { Housework) }\end{array}$ & $\begin{array}{c}2 \\
(10-20 \% \text { of } \\
\text { Housework) } \\
\end{array}$ & $\begin{array}{c}3 \\
(20-30 \% \text { of } \\
\text { Housework) } \\
\end{array}$ & $\begin{array}{c}4 \\
(30-40 \% \text { of } \\
\text { Housework) } \\
\end{array}$ & $\begin{array}{c}5 \\
\text { (40-50\% of } \\
\text { Housework) } \\
\end{array}$ & $\begin{array}{c}6 \\
\text { (50-60\% of } \\
\text { Housework) } \\
\end{array}$ & $\begin{array}{c}7 \\
\text { (60-70\% of } \\
\text { Housework) } \\
\end{array}$ & $\begin{array}{c}8 \\
\text { (70-80\% of } \\
\text { Housework) } \\
\end{array}$ & $\begin{array}{c}9 \\
\text { (80-90\% of } \\
\text { Housework) } \\
\end{array}$ \\
\hline Education & $\begin{array}{l}-0.00376^{*} \\
(0.00204)\end{array}$ & $\begin{array}{l}-0.00398 \\
(0.00366)\end{array}$ & $\begin{array}{l}-0.00253 \\
(0.00390)\end{array}$ & $\begin{array}{c}0.00514 \\
(0.00397)\end{array}$ & $\begin{array}{c}0.0113 * * * \\
(0.00419)\end{array}$ & $\begin{array}{l}0.0171 * * * \\
(0.00472)\end{array}$ & $\begin{array}{l}0.0224 * * * \\
(0.00500)\end{array}$ & $\begin{array}{c}0.0277 * * * \\
(0.00677)\end{array}$ & $\begin{array}{c}0.0250 * * * \\
(0.00770)\end{array}$ \\
\hline Paid Work & $\begin{array}{c}-0.000594 * * * \\
(2.38 \mathrm{e}-05)\end{array}$ & $\begin{array}{c}-0.00178 * * * \\
(3.83 \mathrm{e}-05)\end{array}$ & $\begin{array}{c}-0.00232 * * * \\
(3.83 \mathrm{e}-05)\end{array}$ & $\begin{array}{c}-0.00263 * * * \\
(3.66 \mathrm{e}-05)\end{array}$ & $\begin{array}{c}-0.00285^{* * *} \\
(3.68 \mathrm{e}-05)\end{array}$ & $\begin{array}{c}-0.00317 * * * \\
(3.97 \mathrm{e}-05)\end{array}$ & $\begin{array}{c}-0.00312 * * * \\
(4.04 \mathrm{e}-05)\end{array}$ & $\begin{array}{c}-0.00370 * * * \\
(5.23 \mathrm{e}-05)\end{array}$ & $\begin{array}{c}-0.00310^{* * *} \\
(5.81 \mathrm{e}-05)\end{array}$ \\
\hline Household Inc & $\begin{array}{c}0.0512 * * * \\
(0.0113)\end{array}$ & $\begin{array}{c}0.155^{* * * *} \\
(0.0205)\end{array}$ & $\begin{array}{l}0.166 * * * \\
(0.0223)\end{array}$ & $\begin{array}{c}0.167 * * * \\
(0.0229)\end{array}$ & $\begin{array}{c}0.161^{* * * *} \\
(0.0245)\end{array}$ & $\begin{array}{l}0.168 * * * \\
(0.0280)\end{array}$ & $\begin{array}{l}0.136 * * * \\
(0.0303)\end{array}$ & $\begin{array}{c}0.132 * * * \\
(0.0409)\end{array}$ & $\begin{array}{c}0.0606 \\
(0.0464)\end{array}$ \\
\hline Personal Inc & $\begin{array}{c}-0.206 * * * \\
(0.0311)\end{array}$ & $\begin{array}{c}-0.634 * * * \\
(0.0541)\end{array}$ & $\begin{array}{c}-0.663 * * * \\
(0.0563)\end{array}$ & $\begin{array}{c}-0.657 * * * \\
(0.0556)\end{array}$ & $\begin{array}{c}-0.610 * * * \\
(0.0564)\end{array}$ & $\begin{array}{c}-0.669 * * * \\
(0.0628)\end{array}$ & $\begin{array}{c}-0.620 * * * \\
(0.0649)\end{array}$ & $\begin{array}{c}-0.612 * * * \\
(0.0831)\end{array}$ & $\begin{array}{c}-0.501 * * * \\
(0.0904)\end{array}$ \\
\hline Weekday & $\begin{array}{c}0.0779 * * * \\
(0.00788)\end{array}$ & $\begin{array}{c}0.199 * * * \\
(0.0140)\end{array}$ & $\begin{array}{c}0.244 * * * \\
(0.0148)\end{array}$ & $\begin{array}{c}0.285^{* * * *} \\
(0.0149)\end{array}$ & $\begin{array}{c}0.303 * * * \\
(0.0157)\end{array}$ & $\begin{array}{c}0.344 * * * \\
(0.0178)\end{array}$ & $\begin{array}{c}0.373 * * * \\
(0.0191)\end{array}$ & $\begin{array}{c}0.476 * * * \\
(0.0259)\end{array}$ & $\begin{array}{c}0.455 * * * \\
(0.0300)\end{array}$ \\
\hline Age & $\begin{array}{c}0.00881 * * * \\
(0.000608)\end{array}$ & $\begin{array}{l}0.0213 * * * \\
(0.000992)\end{array}$ & $\begin{array}{c}0.0235 * * * \\
(0.00102)\end{array}$ & $\begin{array}{l}0.0245 * * * \\
(0.000994)\end{array}$ & $\begin{array}{c}0.0261 * * * \\
(0.00101)\end{array}$ & $\begin{array}{l}0.0267 * * * \\
(0.00111)\end{array}$ & $\begin{array}{l}0.0256 * * * \\
(0.00113)\end{array}$ & $\begin{array}{c}0.0291 * * * \\
(0.00146)\end{array}$ & $\begin{array}{c}0.0224 * * * \\
(0.00160)\end{array}$ \\
\hline Household Size & $\begin{array}{c}-0.0106 \\
(0.00774)\end{array}$ & $\begin{array}{l}-0.0129 \\
(0.0137)\end{array}$ & $\begin{array}{l}-0.0199 \\
(0.0148)\end{array}$ & $\begin{array}{l}-0.0113 \\
(0.0151)\end{array}$ & $\begin{array}{l}-0.00817 \\
(0.0159)\end{array}$ & $\begin{array}{l}-0.0138 \\
(0.0178)\end{array}$ & $\begin{array}{l}-0.0113 \\
(0.0189)\end{array}$ & $\begin{array}{c}0.0197 \\
(0.0256)\end{array}$ & $\begin{array}{c}0.0433 \\
(0.0299)\end{array}$ \\
\hline Owns Home & $\begin{array}{c}0.0467 * * * \\
(0.0121)\end{array}$ & $\begin{array}{c}0.106 * * * \\
(0.0207)\end{array}$ & $\begin{array}{c}0.128 * * * \\
(0.0218)\end{array}$ & $\begin{array}{c}0.120 * * * \\
(0.0217)\end{array}$ & $\begin{array}{c}0.117 * * * \\
(0.0225)\end{array}$ & $\begin{array}{c}0.138 * * * \\
(0.0251)\end{array}$ & $\begin{array}{c}0.131 * * * \\
(0.0261)\end{array}$ & $\begin{array}{c}0.147 * * * \\
(0.0340)\end{array}$ & $\begin{array}{c}0.0559 \\
(0.0378)\end{array}$ \\
\hline Employed & $\begin{array}{c}-0.0522 * * * \\
(0.00987)\end{array}$ & $\begin{array}{c}-0.162 * * * \\
(0.0179)\end{array}$ & $\begin{array}{c}-0.218 * * * \\
(0.0194)\end{array}$ & $\begin{array}{c}-0.245^{* * * *} \\
(0.0195)\end{array}$ & $\begin{array}{c}-0.270 * * * \\
(0.0203)\end{array}$ & $\begin{array}{c}-0.273 * * * \\
(0.0224)\end{array}$ & $\begin{array}{c}-0.227 * * * \\
(0.0231)\end{array}$ & $\begin{array}{c}-0.249 * * * \\
(0.0295)\end{array}$ & $\begin{array}{c}-0.176 * * * \\
(0.0318)\end{array}$ \\
\hline Urban & $\begin{array}{l}-0.00910 \\
(0.0107)\end{array}$ & $\begin{array}{l}-0.0376^{*} \\
(0.0192)\end{array}$ & $\begin{array}{l}-0.0400^{*} \\
(0.0205)\end{array}$ & $\begin{array}{l}-0.0292 \\
(0.0206)\end{array}$ & $\begin{array}{c}-0.0437 * * \\
(0.0216)\end{array}$ & $\begin{array}{l}-0.0395 \\
(0.0243)\end{array}$ & $\begin{array}{l}-0.0486^{*} \\
(0.0257)\end{array}$ & $\begin{array}{c}-0.0875 * * \\
(0.0344)\end{array}$ & $\begin{array}{c}-0.119 * * * \\
(0.0394)\end{array}$ \\
\hline Year & $\begin{array}{c}-0.00274 * \\
(0.00146)\end{array}$ & $\begin{array}{c}-0.00921 * * * \\
(0.00260)\end{array}$ & $\begin{array}{c}-0.00894 * * * \\
(0.00279)\end{array}$ & $\begin{array}{c}-0.00858 * * * \\
(0.00284)\end{array}$ & $\begin{array}{c}-0.00905 * * * \\
(0.00299)\end{array}$ & $\begin{array}{c}-0.0109 * * * \\
(0.00338)\end{array}$ & $\begin{array}{c}-0.00781 * * \\
(0.00359)\end{array}$ & $\begin{array}{c}-0.00977 * * \\
(0.00477)\end{array}$ & $\begin{array}{c}0.00136 \\
(0.00537)\end{array}$ \\
\hline \# adults & $\begin{array}{c}0.0388 * * * \\
(0.00858)\end{array}$ & $\begin{array}{c}0.0983 * * * \\
(0.0156)\end{array}$ & $\begin{array}{c}0.129 * * * \\
(0.0169)\end{array}$ & $\begin{array}{c}0.138 * * * \\
(0.0174)\end{array}$ & $\begin{array}{c}0.145^{* * *} \\
(0.0184)\end{array}$ & $\begin{array}{l}0.169 * * * \\
(0.0206)\end{array}$ & $\begin{array}{c}0.167 * * * \\
(0.0220)\end{array}$ & $\begin{array}{l}0.170 * * * \\
(0.0298)\end{array}$ & $\begin{array}{c}0.145 * * * \\
(0.0350)\end{array}$ \\
\hline Constant & $\begin{array}{c}65.19 * * * \\
(2.929)\end{array}$ & $\begin{array}{c}122.5 * * * \\
(5.238)\end{array}$ & $\begin{array}{c}151.8 * * * \\
(5.616)\end{array}$ & $\begin{array}{c}180.9 * * * \\
(5.704)\end{array}$ & $\begin{array}{c}211.8^{* * * *} \\
(6.020)\end{array}$ & $\begin{array}{c}245.4 * * * \\
(6.794)\end{array}$ & $\begin{array}{c}284.1 * * * \\
(7.214)\end{array}$ & $\begin{array}{c}332.9^{* * * *} \\
(9.594)\end{array}$ & $\begin{array}{c}385.9 * * * \\
(10.80)\end{array}$ \\
\hline $\begin{array}{l}\text { Observations } \\
\text { R-squared }\end{array}$ & $\begin{array}{c}45,791 \\
0.053\end{array}$ & $\begin{array}{c}45,791 \\
0.127\end{array}$ & $\begin{array}{c}45,791 \\
0.165\end{array}$ & $\begin{array}{c}45,791 \\
0.190\end{array}$ & $\begin{array}{c}45,791 \\
0.194\end{array}$ & $\begin{array}{c}45,791 \\
0.185\end{array}$ & $\begin{array}{c}45,791 \\
0.157\end{array}$ & $\begin{array}{c}45,791 \\
0.124\end{array}$ & $\begin{array}{c}45,791 \\
0.071\end{array}$ \\
\hline
\end{tabular}


Table A3 UQR Coefficients, Japanese Married Women with Children, STULA 2011 and 2016

\begin{tabular}{|c|c|c|c|c|c|c|c|c|c|}
\hline & $\begin{array}{c}1 \\
(0-10 \% \text { of } \\
\text { Housework) }\end{array}$ & $\begin{array}{c}2 \\
(10-20 \% \text { of } \\
\text { Housework) }\end{array}$ & $\begin{array}{c}3 \\
(20-30 \% \text { of } \\
\text { Housework) } \\
\end{array}$ & $\begin{array}{c}4 \\
(30-40 \% \text { of } \\
\text { Housework) } \\
\end{array}$ & $\begin{array}{c}5 \\
\text { (40-50\% of } \\
\text { Housework) } \\
\end{array}$ & $\begin{array}{c}6 \\
\text { (50-60\% of } \\
\text { Housework) } \\
\end{array}$ & $\begin{array}{c}7 \\
\text { (60-70\% of } \\
\text { Housework) } \\
\end{array}$ & $\begin{array}{c}8 \\
\text { (70-80\% of } \\
\text { Housework) } \\
\end{array}$ & $\begin{array}{c}9 \\
\text { (80-90\% of } \\
\text { Housework) } \\
\end{array}$ \\
\hline Education & $\begin{array}{l}-0.000307 \\
(0.00366)\end{array}$ & $\begin{array}{l}0.000780 \\
(0.00249)\end{array}$ & $\begin{array}{c}0.00277 \\
(0.00276)\end{array}$ & $\begin{array}{l}0.00519 * \\
(0.00275)\end{array}$ & $\begin{array}{c}0.00307 \\
(0.00313)\end{array}$ & $\begin{array}{c}0.00279 \\
(0.00395)\end{array}$ & $\begin{array}{c}0.00200 \\
(0.00450)\end{array}$ & $\begin{array}{c}0.00453 \\
(0.00470)\end{array}$ & $\begin{array}{r}-0.000915 \\
(0.00637)\end{array}$ \\
\hline Paid Work & $\begin{array}{c}-0.00146 * * * \\
(4.77 \mathrm{e}-05)\end{array}$ & $\begin{array}{c}-0.00142 * * * \\
(2.98 \mathrm{e}-05)\end{array}$ & $\begin{array}{c}-0.00181 * * * \\
(3.09 \mathrm{e}-05)\end{array}$ & $\begin{array}{c}-0.00200 * * * \\
(2.87 \mathrm{e}-05)\end{array}$ & $\begin{array}{c}-0.00237 * * * \\
(3.13 \mathrm{e}-05)\end{array}$ & $\begin{array}{c}-0.00290 * * * \\
(3.74 \mathrm{e}-05)\end{array}$ & $\begin{array}{c}-0.00312 * * * \\
(4.16 \mathrm{e}-05)\end{array}$ & $\begin{array}{c}-0.00295^{* * *} \\
(4.28 \mathrm{e}-05)\end{array}$ & $\begin{array}{c}-0.00296^{* * *} \\
(5.71 \mathrm{e}-05)\end{array}$ \\
\hline Household Inc & $\begin{array}{l}0.0540 * * \\
(0.0232)\end{array}$ & $\begin{array}{c}0.0842 * * * \\
(0.0162)\end{array}$ & $\begin{array}{l}0.102 * * * \\
(0.0183)\end{array}$ & $\begin{array}{l}0.127 * * * \\
(0.0184)\end{array}$ & $\begin{array}{c}0.155^{* * * *} \\
(0.0214)\end{array}$ & $\begin{array}{c}0.184 * * * \\
(0.0278)\end{array}$ & $\begin{array}{l}0.193 * * * \\
(0.0322)\end{array}$ & $\begin{array}{l}0.206 * * * \\
(0.0347)\end{array}$ & $\begin{array}{c}0.223 * * * \\
(0.0500)\end{array}$ \\
\hline Personal Inc & $\begin{array}{c}-0.417 * * * \\
(0.0641)\end{array}$ & $\begin{array}{c}-0.508 * * * \\
(0.0432)\end{array}$ & $\begin{array}{c}-0.664 * * * \\
(0.0470)\end{array}$ & $\begin{array}{c}-0.667 * * * \\
(0.0457)\end{array}$ & $\begin{array}{c}-0.748 * * * \\
(0.0517)\end{array}$ & $\begin{array}{c}-0.789 * * * \\
(0.0639)\end{array}$ & $\begin{array}{c}-0.791 * * * \\
(0.0724)\end{array}$ & $\begin{array}{c}-0.698 * * * \\
(0.0753)\end{array}$ & $\begin{array}{c}-0.740 * * * \\
(0.103)\end{array}$ \\
\hline Weekday & $\begin{array}{c}0.287 * * * \\
(0.0145)\end{array}$ & $\begin{array}{l}0.265 * * * \\
(0.00970)\end{array}$ & $\begin{array}{c}0.327 * * * \\
(0.0107)\end{array}$ & $\begin{array}{c}0.348 * * * \\
(0.0106)\end{array}$ & $\begin{array}{c}0.393 * * * \\
(0.0122)\end{array}$ & $\begin{array}{c}0.500 * * * \\
(0.0158)\end{array}$ & $\begin{array}{c}0.561 * * * \\
(0.0182)\end{array}$ & $\begin{array}{c}0.547 * * * \\
(0.0196)\end{array}$ & $\begin{array}{c}0.561 * * * \\
(0.0281)\end{array}$ \\
\hline Age & $\begin{array}{c}0.0295 * * * \\
(0.00109)\end{array}$ & $\begin{array}{l}0.0264 * * * \\
(0.000723)\end{array}$ & $\begin{array}{l}0.0344 * * * \\
(0.000788)\end{array}$ & $\begin{array}{l}0.0375 * * * \\
(0.000766)\end{array}$ & $\begin{array}{l}0.0460 * * * \\
(0.000864)\end{array}$ & $\begin{array}{l}0.0589 * * * \\
(0.00108)\end{array}$ & $\begin{array}{l}0.0656 * * * \\
(0.00122)\end{array}$ & $\begin{array}{c}0.0644 * * * \\
(0.00129)\end{array}$ & $\begin{array}{c}0.0675 * * * \\
(0.00182)\end{array}$ \\
\hline Household Size & $\begin{array}{l}0.102 * * * \\
(0.00851)\end{array}$ & $\begin{array}{l}0.100 * * * \\
(0.00621)\end{array}$ & $\begin{array}{l}0.131 * * * \\
(0.00714)\end{array}$ & $\begin{array}{l}0.150 * * * \\
(0.00731)\end{array}$ & $\begin{array}{l}0.180 * * * \\
(0.00837)\end{array}$ & $\begin{array}{c}0.225^{* * *} \\
(0.0103)\end{array}$ & $\begin{array}{c}0.239 * * * \\
(0.0112)\end{array}$ & $\begin{array}{c}0.235^{* * *} \\
(0.0128)\end{array}$ & $\begin{array}{c}0.263 * * * \\
(0.0183)\end{array}$ \\
\hline Owns Home & $\begin{array}{c}0.0433 * * * \\
(0.0163)\end{array}$ & $\begin{array}{c}0.0324 * * * \\
(0.0113)\end{array}$ & $\begin{array}{l}0.0246^{*} \\
(0.0126)\end{array}$ & $\begin{array}{c}0.0295 * * \\
(0.0126)\end{array}$ & $\begin{array}{c}0.0192 \\
(0.0144)\end{array}$ & $\begin{array}{c}0.0386 * * \\
(0.0181)\end{array}$ & $\begin{array}{l}0.0337 * \\
(0.0205)\end{array}$ & $\begin{array}{c}0.0315 \\
(0.0214)\end{array}$ & $\begin{array}{c}0.0784 * * * \\
(0.0287)\end{array}$ \\
\hline Employed & $\begin{array}{c}-0.128 * * * \\
(0.0193)\end{array}$ & $\begin{array}{c}-0.119 * * * \\
(0.0132)\end{array}$ & $\begin{array}{c}-0.140 * * * \\
(0.0146)\end{array}$ & $\begin{array}{c}-0.151^{* * * *} \\
(0.0144)\end{array}$ & $\begin{array}{c}-0.180 * * * \\
(0.0162)\end{array}$ & $\begin{array}{c}-0.235 * * * \\
(0.0198)\end{array}$ & $\begin{array}{c}-0.248 * * * \\
(0.0221)\end{array}$ & $\begin{array}{c}-0.226 * * * \\
(0.0225)\end{array}$ & $\begin{array}{c}-0.209 * * * \\
(0.0293)\end{array}$ \\
\hline Urban & $\begin{array}{c}-0.0437 * * \\
(0.0204)\end{array}$ & $\begin{array}{c}-0.0302 * * \\
(0.0141)\end{array}$ & $\begin{array}{c}-0.0327 * * \\
(0.0157)\end{array}$ & $\begin{array}{l}-0.0269^{*} \\
(0.0155)\end{array}$ & $\begin{array}{c}-0.0420 * * \\
(0.0176)\end{array}$ & $\begin{array}{l}-0.0194 \\
(0.0221)\end{array}$ & $\begin{array}{l}-0.0275 \\
(0.0250)\end{array}$ & $\begin{array}{l}-0.0133 \\
(0.0261)\end{array}$ & $\begin{array}{l}-0.0350 \\
(0.0358)\end{array}$ \\
\hline Year & $\begin{array}{c}-0.0171 * * * \\
(0.00262)\end{array}$ & $\begin{array}{c}-0.0149 * * * \\
(0.00180)\end{array}$ & $\begin{array}{c}-0.0157 * * * \\
(0.00201)\end{array}$ & $\begin{array}{c}-0.0157 * * * \\
(0.00199)\end{array}$ & $\begin{array}{c}-0.0178 * * * \\
(0.00229)\end{array}$ & $\begin{array}{c}-0.0154 * * * \\
(0.00289)\end{array}$ & $\begin{array}{c}-0.0145 * * * \\
(0.00329)\end{array}$ & $\begin{array}{c}-0.00853 * * \\
(0.00346)\end{array}$ & $\begin{array}{c}0.00705 \\
(0.00478)\end{array}$ \\
\hline \# adults & $\begin{array}{c}-0.179 * * * \\
(0.0116)\end{array}$ & $\begin{array}{c}-0.152 * * * \\
(0.00807)\end{array}$ & $\begin{array}{c}-0.193 * * * \\
(0.00912)\end{array}$ & $\begin{array}{c}-0.198 * * * \\
(0.00914)\end{array}$ & $\begin{array}{c}-0.216^{* * * *} \\
(0.0105)\end{array}$ & $\begin{array}{c}-0.245^{* * * *} \\
(0.0130)\end{array}$ & $\begin{array}{c}-0.242 * * * \\
(0.0145)\end{array}$ & $\begin{array}{c}-0.231 * * * \\
(0.0160)\end{array}$ & $\begin{array}{c}-0.231 * * * \\
(0.0227)\end{array}$ \\
\hline Constant & $\begin{array}{c}108.4 * * * \\
(5.261)\end{array}$ & $\begin{array}{c}149.0 * * * \\
(3.631)\end{array}$ & $\begin{array}{c}180.4 * * * \\
(4.045)\end{array}$ & $\begin{array}{c}210.1 * * * \\
(4.013)\end{array}$ & $\begin{array}{c}244.1 * * * \\
(4.601)\end{array}$ & $\begin{array}{c}283.5 * * * \\
(5.816)\end{array}$ & $\begin{array}{c}311.3 * * * \\
(6.613)\end{array}$ & $\begin{array}{c}344.4 * * * \\
(6.963)\end{array}$ & $\begin{array}{c}402.7 * * * \\
(9.617)\end{array}$ \\
\hline $\begin{array}{l}\text { Observations } \\
\text { R-squared }\end{array}$ & $\begin{array}{c}61,434 \\
0.055 \\
\end{array}$ & $\begin{array}{c}61,434 \\
0.101 \\
\end{array}$ & $\begin{array}{c}61,434 \\
0.128 \\
\end{array}$ & $\begin{array}{c}61,434 \\
0.149 \\
\end{array}$ & $\begin{array}{c}61,434 \\
0.157 \\
\end{array}$ & $\begin{array}{c}61,434 \\
0.149 \\
\end{array}$ & $\begin{array}{c}61,434 \\
0.136 \\
\end{array}$ & $\begin{array}{c}61,434 \\
0.114 \\
\end{array}$ & $\begin{array}{c}61,434 \\
0.066 \\
\end{array}$ \\
\hline
\end{tabular}

